\title{
Algebraic/transcendental disturbance growth behind a row of roughness elements
}

\author{
M. E. GOLDSTEIN ${ }^{1} \dagger$, ADRIAN SESCU ${ }^{2}$, PETER W. DUCK \\ AND MEELAN CHOUDHARI \\ ${ }^{1}$ National Aeronautics and Space Administration, Glenn Research Center, Cleveland, OH 44135, USA \\ ${ }^{2}$ Department of Mechanical Industrial and Manufacturing Engineering, University of Toledo, \\ Toledo, OH 43606, USA \\ ${ }^{3}$ School of Mathematics, University of Manchester, Manchester M13 9PL, UK \\ ${ }^{4}$ National Aeronautics and Space Administration, Langley Research Center, \\ Hampton, VA 23681, USA
}

(Received 25 February 2010; revised 3 September 2010; accepted 8 September 2010)

This paper is a continuation of the work begun in Goldstein et al. (J. Fluid Mech., vol. 644, 2010, p. 123), who constructed an asymptotic high-Reynolds-number solution for the flow over a spanwise periodic array of relatively small roughness elements with (spanwise) separation and plan form dimensions of the order of the local boundary-layer thickness. While that paper concentrated on the linear problem, here the focus is on the case where the flow is nonlinear in the immediate vicinity of the roughness with emphasis on the intermediate wake region corresponding to streamwise distances that are large in comparison with the roughness dimension, but small in comparison with the distance between the roughness array and the leading edge. An analytical $O\left(h^{2}\right)$ asymptotic solution is obtained for the limiting case of a small roughness height parameter $h$. These weakly nonlinear results show that the spanwise variable component of the wall-pressure perturbation decays as $x^{-5 / 3} \ln x$ when $x \rightarrow \infty$ (where $x$ denotes the streamwise distance scaled on the roughness dimension), but the corresponding component of the streamwise velocity perturbation (i.e. the wake velocity) exhibits an $O\left(x^{1 / 3} \ln x\right)$ algebraic/transcendental growth in the main boundary layer. Numerical solutions for $h=O(1)$ demonstrate that the wake velocity perturbation for the fully nonlinear case grows in the same manner as the weakly nonlinear prediction - which is considerably different from the strictly linear result obtained in Goldstein et al. (2010).

Key words: boundary-layer receptivity, boundary-layer stability, boundary-layer structure

\section{Introduction}

It is suspected that roughness-induced streaks play an important role in the bypass transition due to distributed, three-dimensional surface roughness in boundary-layer flows that are linearly stable or, at most, weakly unstable. In fact, it is well known that certain types of streak-like perturbations may undergo a transient algebraic 
(a)

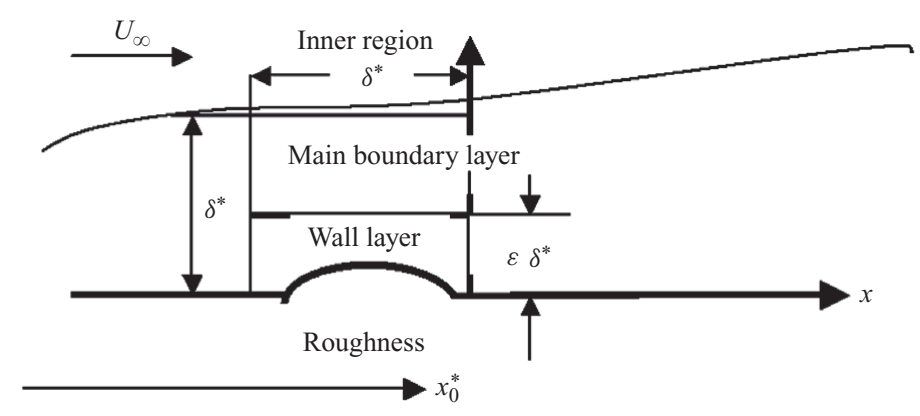

(b)

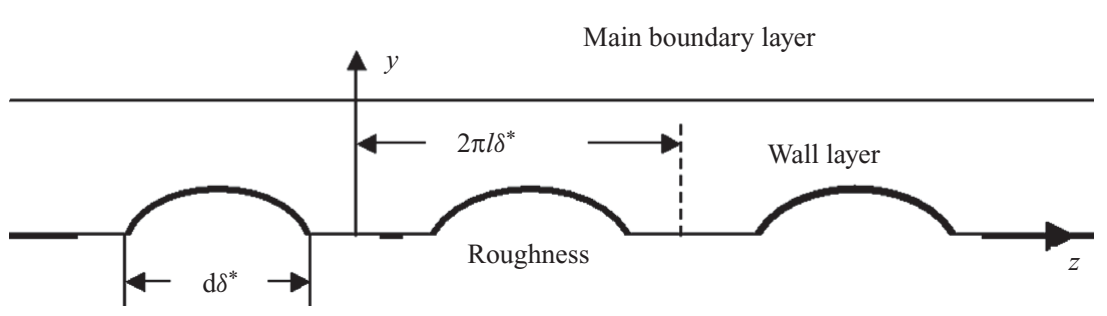

FIGURE 1. Boundary-layer flow structure, $(a)$ side view, $(b)$ cross-sectional view.

growth prior to an eventual exponential decay in linearly stable shear flows (Case 1960; Ellingson \& Palm 1975; Landahl 1980). It is also known that streaks can have a stabilizing effect on the small amplitude Tollmien-Schlichting waves that comprise the initial stage of transition in many applications with low levels of external forcing. Moreover it is now realized that the stable laminar streaks generated by surface roughness elements can be used to control transition in these applications (Fransson et al. 2004, 2006). It therefore seems important to understand the physical mechanisms related to the potential disturbance growth in the wake flow behind surface roughness elements.

It is generally believed that algebraic or non-modal growth arises from the "liftup' effect associated with the spanwise varying displacement of a two-dimensional shear flow (Landahl 1980). This phenomenon is typically found to take place over streamwise length scales that are comparable to the downstream distance from the leading edge (e.g. Andersson, Berggren \& Henningson 1999), but the present paper shows that transcendental (algebraic/logarithmic) growth can also occur on a much shorter streamwise length scale, which is still large compared to the roughness elements themselves, but small compared to the distance from the leading edge.

The analysis, which continues the work begun in Goldstein et al. (2010, hereafter referred to as GSDC), is based on an asymptotic high-Reynolds-number solution for the flow over a spanwise periodic array of relatively small roughness elements whose (spanwise) separation and plan form dimensions are of the order of the local boundary-layer thickness $\delta^{*}$ (figure 1). An analogous two-dimensional problem was studied in considerable detail by Smith (1973) (see also Smith 1976a, b; Smith et al. 1981; Rothmayer \& Smith 1998). The GSDC result shows that the local flow in the vicinity of the roughness has a double layer structure with the same scaling as was used in Choudhari \& Duck (1996). However, the solution becomes invalid 
in the far-wake region, where non-parallel effects come into play over downstream distances comparable to the distance from the leading edge. While GSDC present an asymptotic framework for a broad range of roughness heights and include preliminary results for the case where the near-field perturbations are nonlinear functions of the roughness height, the main focus of that paper was on the limiting case of small height parameters.

In contrast, the present paper focuses on the case where the flow is nonlinear in the vicinity of the roughness, with emphasis on the intermediate region that lies far downstream from the roughness (compared to the roughness dimensions), but much closer than the downstream distance $x_{0}^{*}$ between the roughness and the leading edge. The flow in this region is still governed by a linear set of equations that has an exact similarity solution when the spanwise variable component of the wall pressure decays as $x^{-\alpha}$, where $x$ denotes the streamwise distance behind the roughness elements scaled on the dimension of the roughness. But (as noted at the beginning of the discussion in GSDC) this same set of equations has also an asymptotic solution when the wall pressure decay has the more general algebraic/transcendental form $x^{-\alpha}(\ln x)^{\beta}$, with $\alpha>0, \beta \geqslant 0$. GSDC's exact analytical solution for the strictly linear problem (where the scaled roughness height $h \ll 1$ ) exhibited purely algebraic decay with the decay rate exponent $\alpha$ being equal to $8 / 3$, while their numerical solutions for the fully nonlinear case seemed to be consistent with an $x^{-5 / 3}$ algebraic decay (i.e. $\alpha=5 / 3, \beta=0$ ). The present paper shows that the spanwise variable wall pressure actually behaves transcendentally and decays at the slightly slower $x^{-5 / 3} \ln x$ rate (i.e. $\alpha=5 / 3, \beta=1)$. This is accomplished by carrying the small $h$ asymptotic solution to second order (i.e. to $O\left(h^{2}\right)$ ) and comparing the result with full $h=O(1)$ numerical solutions.

The strictly linear solution obtained in GSDC shows that the peak value of the streamwise component of the spanwise variable wake velocity defect decays as $1 / x$ in the downstream wall layer, but the present result shows that it actually increases as $\ln x$ when $x \rightarrow \infty$ in the fully nonlinear case. This does not, however, destroy the linearity of the downstream flow because the peak velocity defect moves upward into the main boundary layer as $x^{1 / 3}$ when $x \rightarrow \infty$, which means that the local Blasius velocity (about which the equations are linearized) also increases as $x^{1 / 3}$ and, therefore, always remains larger than the wake velocity defect.

This increase in spanwise variable streamwise velocity perturbation, which is caused by the interaction between the various spanwise Fourier modes in the nonlinear case, produces an effect that is similar to the well-known (Landahl 1980) 'lift-up' effect, which is generated by a purely linear mechanism and is probably responsible for the transient growth that occurs in the strictly linear case considered in GSDC. The present mechanism does not occur in the linear case because these modal interactions do not occur in that situation. It is worth noting that the $O(h)$ and $O\left(h^{2}\right)$ streamwise velocity components decay at the same rate in the analogous two-dimensional problem studied by Smith (1973) and Smith et al. (1981) where the spanwise modal interactions cannot occur - which reinforces the notion that the velocity defect amplification is associated with the interaction between the spanwise modes in the present case. The numerical computations show that the dominant contribution to the nonlinear wall pressure comes from the interaction between the fundamental spanwise harmonic and spanwise mean of the perturbation velocity component. So most of the nonlinear growth in the streamwise velocity is caused by a 'linear' interaction between the fundamental spanwise mode and the roughness induced two-dimensional (spanwise mean) flow distortion. This is not too different from what happens in the linear 
case where most of the algebraic growth results from the interaction between the fundamental harmonic and the two-dimensional boundary-layer flow resulting from the slow growth of the boundary layer over the viscous length scale.

The streamwise velocity perturbation eventually moves up into the main boundary layer or main deck, where the Blasius velocity is more uniform and the spanwise variable streamwise velocity now increases at a much more rapid $x^{1 / 3} \ln x$ rate. The spanwise variable wake velocity, therefore, exhibits algebraic/transcendental growth in the fully nonlinear case, while the GSDC result shows that it decays as $x^{-2 / 3}$ in the strictly linear case.

The GSDC analysis can be extended to show that this intermediate scale solution eventually breaks down and the flow evolves on the much longer scale over which the unperturbed boundary layer develops. The solution in this far-wake region, which brings in the non-parallel mean flow effects and is governed by the boundary region equations of Kemp (1951), is nearly identical to the result given in GSDC, but the more rapid transcendental growth over the intermediate region causes the spanwise varying component of streamwise velocity perturbation in the far wake to be larger than the GSDC result by a factor of $\ln \left(x_{0}^{*} / \delta^{*}\right)^{1 / 3}$ when the flow in the vicinity of the roughness is fully nonlinear. The wake velocity defect initially follows the algebraic/transcendental growth that it developed in the intermediate region, but non-parallel flow effects cause it to saturate and eventually decay. In other words, it exhibits transient growth on this longer streamwise length scale. This is in stark contrast to the strictly linear result discussed in GSDC, where the wake velocity defect decays on the intermediate length scale and begins to grow only at downstream distances of the order of the boundary-layer growth scale.

The problem is formulated in $\S 2$ and the local asymptotic high-Reynolds-number solution in the vicinity of the roughness, which has the same scaling as in Choudhari \& Duck (1996), is outlined in $\S 3$. Section 4 is concerned with the far downstream (on the roughness scale $\delta^{*}$ ) region where the flow becomes linear. The $O\left(h^{2}\right)$ asymptotic small roughness height solution is derived in $\S 5$ and compared with the $h=O(1)$ numerical solution in $\S 6$. Conclusions are presented in $\S 7$.

\section{Problem formulation and asymptotic scaling equation}

As in GSDC, we consider an incompressible flat-plate boundary layer that is perturbed by a spanwise periodic linear array of roughness elements and suppose that the spanwise wavelength of the roughness array, say $2 \pi l \delta^{*}$, is comparable to the local value of the boundary-layer similarity length scale $\delta^{*} \equiv x_{0}^{*} / \sqrt{R}=x_{0}^{*} \delta$ at the roughness location $x^{*}=x_{0}^{*}$, where $R \equiv x_{0}^{*} U_{\infty} / \nu^{*}$ is the Reynolds number based on $x_{0}^{*}$ and the free-stream velocity $U_{\infty}$ with $v^{*}$ being the kinematic viscosity and $\delta \equiv R^{-1 / 2} \ll 1$ being the scaled boundary-layer thickness. The roughness elements are assumed to have more or less circular cross-section with the same streamwise and spanwise scaling and to be small enough to produce only local separation. We therefore write

$$
\begin{gathered}
y=y_{r}=\varepsilon h \tilde{F}(x, z), \\
u\left(x, y_{r}, z\right)=v\left(x, y_{r}, z\right)=w\left(x, y_{r}, z\right)=0,
\end{gathered}
$$

where all lengths are also normalized with respect to the boundary-layer thickness at $x^{*}=x_{0}^{*}$ unless otherwise noted, $y_{r}$ is the roughness height, $\varepsilon \equiv R^{-1 / 6}=\delta^{1 / 3} \ll 1$, 
$x \equiv\left(x^{*}-x_{0}^{*}\right) / \delta^{*}$ and, as usual, the fluid velocity

$$
\boldsymbol{v}=\{u, v, w\}
$$

is normalized by $U_{\infty}$ and the pressure $p$ by $\rho U_{\infty}^{2}$, where $\rho$ is the fluid density.

\section{Asymptotic structure of inner solution and governing equations}

\subsection{Main boundary-layer region}

GSDC divide the boundary-layer flow into an inner region in the vicinity of the roughness elements and an outer region that lies further downstream. The focus of the present paper is on the former region where the asymptotic scaling is essentially the same as that used in Choudhari \& Duck (1996). The solution in the main boundary layer, where $y=O(1)$, therefore expands as

$$
\{u, v, w, p\}=\left\{U_{B}, \varepsilon^{3} V_{B}, 0,0\right\}+\varepsilon^{2}\left\{u_{0}, v_{0}, w_{0}, p_{0}\right\}+\cdots,
$$

where

$$
U_{B}(y)=F^{\prime}(y) \rightarrow \lambda y+O\left(y^{4}\right) \text { as } y \rightarrow 0
$$

is the Blasius velocity with the Blasius function $F$ being determined from the Blasius equation in the usual way and $\lambda=0.33206$. GSDC show that the $O\left(\varepsilon^{2}\right)$ solution $\left\{u_{0}, v_{0}, w_{0}, p_{0}\right\}$, is determined by

$$
\begin{gathered}
U_{B}(y) \frac{\partial^{2} u_{0}}{\partial x^{2}}=\left[U_{B}^{\prime}(y) / U_{B}(y)\right] \frac{\partial p_{0}}{\partial y}-\frac{\partial^{2} p_{0}}{\partial x^{2}}, \\
U_{B}(y) \frac{\partial w_{0}}{\partial x}=-\frac{\partial p_{0}}{\partial z}, \\
\nabla^{2} p_{0}-2 \frac{U_{B}^{\prime}(y)}{U_{B}(y)} \frac{\partial p_{0}}{\partial y}=0,
\end{gathered}
$$

subject to the boundary condition

$$
p_{0}(x, 0, z)=P(x, z)
$$

where the $O\left(\varepsilon^{2}\right)$ wall pressure $P(x, z)$ will be specified more precisely below.

Since the roughness is assumed to be periodic in the spanwise direction and the flow in this region is linear, the spanwise and streamwise velocities possess the Fourier expansions

$$
\begin{aligned}
& w_{0}(x, y, z)=-\frac{1}{U_{B}(y)} \sum_{n=-\infty}^{n=\infty} \int_{-\infty}^{\infty} \frac{n \tilde{P}_{n}(k)}{l k} \pi_{n}(y, k) \mathrm{e}^{\mathrm{i}((n z / l)+k x)} \mathrm{d} k \\
& u_{0}(x, y, z)=-\frac{1}{U_{B}(y)} \sum_{n=-\infty}^{n=\infty} \int_{-\infty}^{\infty} \tilde{P}_{n}(k)\left[\frac{U_{B}^{\prime}(y)}{k^{2} U_{B}(y)} \frac{\mathrm{d} \pi_{n}(y, k)}{\mathrm{d} y}+\pi_{n}(y, k)\right] \mathrm{e}^{\mathrm{i}((n z / l)+k x)} \mathrm{d} k
\end{aligned}
$$

in terms of the solution $\pi_{n}(y, k)$ to the unit boundary value problem

$$
\begin{gathered}
U_{B}^{2}(y) \frac{\mathrm{d}}{\mathrm{d} y}\left[\frac{1}{U_{B}^{2}(y)} \frac{\mathrm{d} \pi_{n}}{\mathrm{~d} y}\right]-\left[\left(\frac{n}{l}\right)^{2}+k^{2}\right] \pi_{n}=0, \quad n=0, \pm 1, \pm 2, \ldots \\
\pi_{n}(0, k)=1, \pi_{n}(y, k) \rightarrow 0 \quad \text { as } y \rightarrow \infty,
\end{gathered}
$$


and the spanwise harmonic Fourier coefficients $\tilde{P}_{n}^{(0)}(k)$ of the wall pressure

$$
P(x, z)=\sum_{n=-\infty}^{n=\infty} \int_{-\infty}^{\infty} \tilde{P}_{n}(k) \mathrm{e}^{\mathrm{i}((n z / l)+k x)} \mathrm{d} k .
$$

Since $y=0$ is a regular singular point of (3.9), it follows from (3.2) and from the method of Frobenius that the two linearly independent solutions of (3.9) have Taylor series expansions about this point and, therefore, that

$$
\pi_{n}(y, k) \sim 1-\frac{1}{2}\left[\left(\frac{n}{l}\right)^{2}+k^{2}\right] y^{2}+O\left(y^{3}\right), \quad \text { as } y \rightarrow 0,
$$

which shows that

$$
u_{0}(x, y, z)=O\left(y^{-1}\right)
$$

and

$$
w_{0}(x, y, z) \rightarrow-\frac{1}{\lambda y} \sum_{n=-\infty}^{n=\infty} \int_{-\infty}^{\infty} \frac{n \tilde{P}_{n}(k)}{l k}\left[1+O\left(y^{2}\right)\right] \mathrm{e}^{\mathrm{i}((n z / l)+k x)} \mathrm{d} k, \quad \text { as } y \rightarrow 0 .
$$

\subsection{Wall-layer region}

The singularity in $u_{0}$ as $y \rightarrow 0$ causes the expansion (3.1) to break down when

$$
\breve{Y} \equiv y / \varepsilon=O(1)
$$

and it is then necessary to obtain a new solution in this region, which we refer to here as the wall layer. Equations (3.13) and (3.14) show that the solution in this region must expand as (see Choudhari \& Duck 1996)

$$
\{u, v, w, p\}=\varepsilon\{U, \varepsilon \bar{V}, W, \varepsilon P(x, z)\}+\cdots,
$$

where we have introduced the Prandtl transformation

$$
\begin{gathered}
Y \equiv y / \varepsilon-h \tilde{F}(x, z)=\breve{Y}-h \tilde{F}(x, z), \\
V \equiv \breve{V}-h\left(\tilde{F}_{x} U+\tilde{F}_{z} W\right),
\end{gathered}
$$

so that all velocity perturbations are functions of $\{x, Y, z\}$, and it is anticipated that the pressure does not vary across this thinner region to the required degree of approximation. The leading-order solution $\{U, V, W, P\}$ is determined from the three-dimensional boundary-layer equations

$$
\begin{aligned}
U_{x}+V_{Y}+W_{z} & =0, \\
U W_{x}+V W_{Y}+W W_{z} & =-P_{z}+W_{Y Y}, \\
U U_{x}+V U_{Y}+W U_{z} & =-P_{x}+U_{Y Y},
\end{aligned}
$$

subject to the boundary conditions

$$
\begin{gathered}
U=V=W=0 \quad \text { at } Y=0, \\
U \rightarrow \lambda Y ; V, W \rightarrow 0, \quad \text { as } x \rightarrow \pm \infty,
\end{gathered}
$$

and the matching condition

$$
W_{x} \rightarrow-P_{z} / \lambda Y, U \rightarrow \lambda(Y+h \tilde{F})+O\left(y^{-1}\right), \quad \text { as } Y \rightarrow \infty .
$$


This system may be regarded as the three-dimensional analogue of the zerodisplacement two-dimensional problem considered by Smith $(1976 a, b)$ even though there is a significant difference in its algebraic decay as $Y \rightarrow \infty$.

\section{Downstream behaviour of solution}

The wall pressure $P(x, z)$ is expected to decay algebraically/transcendentally fast as $x \rightarrow \infty$. However, the GSDC results show that the spanwise mean component $\bar{P}(x)$ of $P(x, z)$ behaves differently from the spanwise variable component and must, therefore, be considered separately. We subtract out the mean component (which is of little interest in the present context because it does not contribute to the threedimensional far-wake flow that is being considered here) and require that $P(x, z)-\bar{P}(x)$ behave as $x^{-\alpha}(\ln x)^{\beta}$ for some real constants $\alpha>0$ and $\beta \geqslant 0$, as $x \rightarrow \infty$. It then follows from (3.6) that

$$
p_{0}(x, y, z)-\bar{p}_{0}(x, y) \sim \hat{p}(y, z)\left(\ln x^{1 / 3}\right)^{\beta} / x^{\alpha}, \quad \text { as } x \rightarrow \infty,
$$

where $\bar{p}_{0}(x, y)$ denotes the spanwise average component of $p_{0}(x, y, z)$ (and similarly for the remaining dependent variables $u_{0}(x, y, z), v_{0}(x, y, z)$ and $\left.w_{0}(x, y, z)\right)$.

The analysis given in Carrier, Krook \& Pearson (1966, pp. 255 and 256) can easily be extended to show that the corresponding Fourier coefficients in (3.11) $\tilde{P}_{n}(k), n \neq 0$ must behave as

$$
\tilde{P}_{n}(k) \sim(\mathrm{i} k)^{\alpha-1}\left(\ln k^{1 / 3}\right)^{\beta} \tilde{a}_{n}, \quad \text { as } k \rightarrow 0, \quad n \neq 0,
$$

where the $\tilde{a}_{n}$ are constants. The Carrier et al. (1966) results also imply that the coefficient $\hat{p}(y, z)$ in (4.1) is related to the unit solutions $\pi_{n}(n= \pm 1, \pm 2, \ldots)$ of the Rayleigh equation, via

$$
\hat{p}(y, z) \equiv-2[\sin \pi(\alpha-1)] \Gamma(\alpha) \sum_{\substack{n=-\infty \\ n \neq 0}}^{n=\infty} \pi_{n}(y, 0) \tilde{a}_{n} \mathrm{e}^{\mathrm{i} n z / l} .
$$

And then the main-deck solutions (3.7) and (3.8) imply that

$$
\begin{aligned}
w_{0}(x, y, z) & \sim \frac{\left(\ln x^{1 / 3}\right)^{\beta}}{(\alpha-1) x^{\alpha-1}} \frac{1}{U_{B}(y)} \frac{\partial \hat{p}(y, z)}{\partial z}, \\
u_{0}(x, y, z)-\bar{u}_{0}(x, y) & \sim \frac{\left(\ln x^{1 / 3}\right)^{\beta}}{(\alpha-1)(\alpha-2) x^{\alpha-2}} \frac{U_{B}^{\prime}(y)}{U_{B}^{2}(y)} \frac{\partial}{\partial y} \hat{p}(y, z),
\end{aligned}
$$

as $x \rightarrow \infty$. Substituting the expansion (3.12) for the near-wall behaviour of the unit solutions $\pi_{n}$ into (4.3) yields

$$
\hat{p}(y, z) \sim-2[\sin \pi(\alpha-1)] \Gamma(\alpha) \sum_{\substack{n=-\infty \\ n \neq 0}}^{n=\infty}\left[1-\frac{1}{2}\left(\frac{n}{l}\right)^{2} y^{2}+O\left(y^{3}\right)\right] \tilde{a}_{n}^{(0)} \mathrm{e}^{\mathrm{i} n z / l}, \quad \text { as } y \rightarrow 0,
$$

and it then follows that

$$
\begin{aligned}
w_{0}(x, y, z) & \sim \frac{\left(\ln x^{1 / 3}\right)^{\beta}}{(\alpha-1) x^{\alpha-1} \lambda y} \frac{\partial \hat{p}(0, z)}{\partial z}, \quad \text { as } y \rightarrow 0, \\
u_{0}(x, y, z)-\bar{u}_{0}(x, y) & \sim \frac{\left(\ln x^{1 / 3}\right)^{\beta}}{(\alpha-1)(\alpha-2) x^{\alpha-2} \lambda y} \frac{\partial^{2} \hat{p}(0, z)}{\partial z^{2}}, \quad \text { as } y \rightarrow 0,
\end{aligned}
$$


which provide the boundary conditions for the asymptotic wall-layer solution far downstream in the flow.

This solution is expected to decay relative to the Blasius velocity, and therefore exhibits linear behaviour when $x \gg 1$, even when it is nonlinear in the vicinity of the roughness, which suggests that $\{U-\lambda Y, V, W, P\}$ should be a small perturbation of the original Blasius flow $U=\lambda Y$, and therefore satisfy the linear equations

$$
\begin{aligned}
\frac{\partial(U-\lambda Y)}{\partial x}+\frac{\mathrm{d} V}{\mathrm{~d} Y}+\frac{\partial W}{\partial z} & =0, \\
\lambda Y \frac{\partial(U-\lambda Y)}{\partial x}+\lambda V+\frac{\partial P}{\partial x} & =\frac{\partial^{2} U}{\partial Y^{2}}, \\
\lambda Y \frac{\partial W}{\partial x}+\frac{\partial P}{\partial z} & =\frac{\partial^{2} W}{\partial Y^{2}},
\end{aligned}
$$

far downstream in the flow.

The spanwise mean and zero-spanwise mean components can, therefore, be considered separately in the large $x$ downstream region, which is important because the main boundary-layer solution shows that they behave differently at large $x$. The results for the spanwise mean component are derived in Appendix A of GSDC, and only the spanwise varying component (which is the one of principal interest) is dealt with here.

As noted in GSDC, these equations possess an exact similarity solution of the form

$$
W=x^{2 / 3-\alpha} \tilde{W}(\eta, z), \quad U-\bar{U}=x^{5 / 3-\alpha} \tilde{U}(\eta, z), \quad V-\bar{V}=x^{1-\alpha} \lambda^{-1 / 3} \tilde{V}(\eta, z),
$$

where

and $\tilde{U}, \tilde{V}, \tilde{W}$ satisfy

$$
\eta \equiv \lambda^{1 / 3} Y / x^{1 / 3}
$$

$$
\begin{aligned}
-\left[\frac{\eta}{3} \frac{\partial \tilde{U}}{\partial \eta}+\left(\alpha-\frac{5}{3}\right) \tilde{U}\right]+\frac{\partial \tilde{V}}{\partial \eta}+\frac{\partial \tilde{W}}{\partial z} & =0 \\
\frac{\partial^{2} \tilde{U}}{\partial \eta^{2}}+\eta\left[\frac{\eta}{3} \frac{\partial \tilde{U}}{\partial \eta}+\left(\alpha-\frac{5}{3}\right) \tilde{U}\right] & =\tilde{V} \\
\frac{\partial^{2} \tilde{W}}{\partial \eta^{2}}+\eta\left[\frac{\eta}{3} \frac{\partial \tilde{W}}{\partial \eta}+\left(\alpha-\frac{2}{3}\right) \tilde{W}\right] & =\lambda^{-2 / 3} \frac{\partial}{\partial z} \hat{p}(0, z),
\end{aligned}
$$

subject to the boundary conditions

$$
\tilde{U}(0, z)=V(0, z)=W(0, z)=0 .
$$

However, it is easy to verify that they also possess an asymptotic solution of the slightly more general form

$$
\begin{aligned}
W & =x^{2 / 3-\alpha}\left(\ln x^{1 / 3}\right)^{\beta} \tilde{W}(\eta, z), \\
U-\bar{U} & =x^{5 / 3-\alpha}\left(\ln x^{1 / 3}\right)^{\beta} \tilde{U}(\eta, z),
\end{aligned}
$$

and

$$
V-\bar{V}=x^{1-\alpha}\left(\ln x^{1 / 3}\right)^{\beta} \lambda^{-1 / 3} \tilde{V}(\eta, z),
$$

to within a relative error of $O(\ln x)^{-1}$ as $x \rightarrow \infty$, where $\tilde{U}, \tilde{V}, \tilde{W}$ again satisfy the similarity equations (4.14)-(4.16), and $\beta$ is an arbitrary positive constant which like $\alpha$ is determined by the upstream boundary conditions, i.e. by the asymptotic behaviour of the local solution in the vicinity of the roughness. GSDC show that it is equal to 
zero in the strictly linear case (i.e. (4.18)-(4.20) reduce to (4.12)) and $\S 5$ shows that it is equal to the one in the weakly nonlinear case (see comments below (5.25)), while the numerical results show that it is also equal to the one in the fully nonlinear case (see discussion of figure 6 below).

Equations (3.1), (3.16) to (3.18), (4.7), (4.8) and (4.18) to (4.20) show that these solutions will match onto the main boundary-layer solution if we require that

$$
\begin{aligned}
& \tilde{W}(\eta, z) \rightarrow \frac{1}{(\alpha-1) \lambda^{2 / 3} \eta} \frac{\partial \hat{p}(0, z)}{\partial z}, \\
& \tilde{U}(\eta, z) \rightarrow \frac{1}{(\alpha-1)(\alpha-2) \lambda^{2 / 3} \eta} \frac{\partial^{2} \hat{p}(0, z)}{\partial z^{2}} .
\end{aligned}
$$

GSDC show that the streamwise velocity component of the solution to (4.14)-(4.16) that satisfies (4.17), (4.21) and (4.22) is given by

$$
\tilde{U}=\frac{1}{\lambda^{2 / 3}(\alpha-1) C} \frac{\partial^{2} \hat{p}(0, z)}{\partial z^{2}}\left[\hat{U}(\eta)+\hat{C}_{H} \hat{U}_{H}(\eta)\right]+\tilde{U}_{H},
$$

where $\hat{C}_{H}, \tilde{B}_{H}$ and $C$ are constants,

$$
\begin{gathered}
\tilde{U}_{H}=\tilde{B}_{H} \int_{0}^{\eta}{ }_{1} F_{1}\left(\frac{3 \alpha-4}{3}, \frac{2}{3} ;-\frac{\eta^{3}}{9}\right) \mathrm{d} \eta \\
-\frac{1}{\lambda^{2 / 3}(\alpha-1)} \frac{\partial^{2} \hat{p}(0, z)}{\partial z^{2}} \int_{0}^{\eta} \eta_{1} F_{1}\left(\frac{3 \alpha-3}{3}, \frac{4}{3} ;-\frac{\eta^{3}}{9}\right) \mathrm{d} \eta \\
\hat{U}_{H}=\eta_{1} F_{1}\left(\frac{3 \alpha-4}{3}, \frac{4}{3} ;-\frac{\eta^{3}}{9}\right),
\end{gathered}
$$

and $\hat{U}(\eta)$ is determined by

$$
\frac{\partial^{2} \hat{U}}{\partial \eta^{2}}+\eta\left[\frac{\eta}{3} \frac{\partial \hat{U}}{\partial \eta}+\left(\alpha-\frac{5}{3}\right) \hat{U}\right]=C,
$$

subject to the boundary conditions

$$
\hat{U}(0)=0 \quad \text { and } \quad \hat{U}(\eta) \rightarrow C /[(\alpha-2) \eta], \quad \text { as } \eta \rightarrow \infty,
$$

provided the integrals in (4.24) converge as $\eta \rightarrow \infty$. Each of them will converge when $\alpha>5 / 3$ (as it is in the strictly linear case considered in GSDC) and $\hat{C}_{H}$ can then be chosen so that the boundary condition (4.22) is satisfied.

However, the combined integral in (4.24) will converge even when $\alpha \leqslant 5 / 3$ if $\tilde{B}_{H}$ is set equal to

$$
\tilde{B}_{H}=\frac{\Gamma(2-\alpha) \Gamma(1 / 3)}{\Gamma(2-\alpha+1 / 3) \Gamma(2 / 3) 3^{1 / 3}} \frac{1}{\lambda^{2 / 3}(\alpha-1)} \frac{\partial^{2} \hat{p}(0, z)}{\partial z^{2}},
$$

because (see 13.1.9 on p. 504 of Abramowitz \& Stegun 1965) the integrand will then be proportional to $\mathrm{e}^{-\eta^{3} / 9} U\left((6-3 \alpha) / 3,2 / 3, \eta^{3} / 9\right.$ ) (where $U(a, b, z)$ denotes the hypergeometric equation solution defined in Abramowitz \& Stegun (1965, equation 13.1.3)) and, therefore, vanishes exponentially fast as $\eta \rightarrow \infty$. It follows that the constant $\hat{C}_{H}$ can be chosen so that

$$
\tilde{U} \rightarrow \frac{1}{\lambda^{2 / 3}(\alpha-1) C} \frac{\partial^{2} \hat{p}(0, z)}{\partial z^{2}} \hat{U}(\eta), \quad \text { as } \eta \rightarrow \infty,
$$


when $\alpha=5 / 3$ and the boundary condition (4.22) can, therefore, also be satisfied in this case. The homogeneous solution (4.25) is given by

$$
\hat{U}_{H}=\int_{0}^{\eta} \exp \left(-\frac{\eta^{3}}{9}\right) \mathrm{d} \eta
$$

for this value of $\alpha$ (i.e. $\alpha=5 / 3$ ) and (4.24) can be expressed in terms of the hypergeometric equation solution $U(a, b, z)$ to obtain

$$
\tilde{U}_{H}=\left(\frac{3}{\lambda}\right)^{2 / 3} \frac{[\Gamma(1 / 3)]^{2} \sqrt{3}}{4 \pi} \frac{\partial^{2} \hat{p}(0, z)}{\partial z^{2}} \int_{0}^{\eta} \mathrm{e}^{-\eta^{3} / 9} U\left(1 / 3,2 / 3, \eta^{3} / 9\right) \mathrm{d} \eta .
$$

Equation (4.19) shows that $U-\bar{U}$ will then increase as $(\ln x)^{\beta}$ when $x \rightarrow \infty$ at fixed $\eta$ and $z$, but the linearization (4.9)-(4.11) will still be valid because the scaled Blasius velocity $U_{B} / \varepsilon \approx \lambda Y=\lambda^{2 / 3} x^{1 / 3} \eta$ increases as $x^{1 / 3}$ when $x \rightarrow \infty$ at fixed $\eta$. However, this is the smallest value of $\alpha$ for which the linearization will be valid, because matching the wall layer and main-deck solutions requires that $\alpha=n / 3$ for $n=1,2, \ldots$ (since the inner expansion of the latter can only involve integral powers of $y$ ) and $U-\bar{U}$ will be proportional to $\left(\ln x^{1 / 3}\right)^{\beta} x^{1 / 3}$ as $x \rightarrow \infty$ at fixed $\eta$ when $\alpha$ is equal to $4 / 3$.

\section{Asymptotic solution for $h \ll 1$}

This section constructs an asymptotic solution (valid in the limit $h \rightarrow 0$ ) in order to fix the decay rate exponents $\alpha$ and $\beta$ and explicitly relate the wall pressure to the roughness geometry.

\subsection{Solution for $x=O(1)$}

When $h \ll 1$, the wall-layer solution possesses the expansions

$$
\begin{aligned}
\{U, V, W, P(x, z)\}=\{\lambda Y, 0.0,0\}+h\{ & \left.U_{1}, V_{1}, W_{1}, P_{1}(x, z)\right\} \\
& +h^{2}\left\{U_{2}, V_{2}, W_{2}, P_{2}(x, z)\right\}+\cdots,
\end{aligned}
$$

where $\left\{U_{j}, V_{j}, W_{j}, P_{j}\right\}$ satisfy the boundary conditions (3.22) and (3.23), the matching condition (3.24) and the linear equations

$$
\begin{array}{r}
\frac{\partial U_{j}}{\partial x}+\frac{\mathrm{d} V_{j}}{\mathrm{~d} Y}+\frac{\partial W_{j}}{\partial z}=0, \\
\lambda Y \frac{\partial U_{j}}{\partial x}+\lambda V_{j}+\frac{\partial P_{j}}{\partial x}-\frac{\partial^{2} U_{j}}{\partial Y^{2}}=F_{j}, \\
\lambda Y \frac{\partial W_{j}}{\partial x}+\frac{\partial P_{j}}{\partial z}-\frac{\partial^{2} W_{j}}{\partial Y^{2}}=G_{j},
\end{array}
$$

where

$$
\begin{gathered}
F_{1}=0, F_{2}=-U_{1} \frac{\partial U_{1}}{\partial x}-V_{1} \frac{\partial U_{1}}{\partial Y}-W_{1} \frac{\partial U_{1}}{\partial z}, \ldots, \\
G_{1}=0, \mathrm{G}_{2}=-U_{1} \frac{\partial W_{1}}{\partial x}-V_{1} \frac{\partial W_{1}}{\partial Y}-W_{1} \frac{\partial W_{1}}{\partial z}, \ldots
\end{gathered}
$$

Equations (3.22)-(3.24) then imply that

$$
\widehat{U}_{n}^{(j)}=\widehat{W}_{n}^{(j)}=\widehat{V}_{n}^{(j)}=0, \quad \text { at } Y=0,
$$


for $j=1,2, \ldots$, while (3.24) implies that

$$
\widehat{U}_{n}^{(1)}=\lambda \widehat{F}_{n}(k)+O\left(Y^{-1}\right), \quad \hat{W}_{n}^{(1)}=O\left(Y^{-1}\right), \quad \text { as } Y \rightarrow \infty,
$$

and

$$
\hat{U}_{n}^{(2)}(k)=O\left(Y^{-1}\right), \quad \widehat{W}_{n}^{(2)}=-\frac{n \tilde{P}_{n}^{(2)}}{k l \lambda Y}, \quad \text { as } Y \rightarrow \infty,
$$

where $\left\{\widehat{U}_{n}^{(j)}(Y, k), \widehat{V}_{n}^{(j)}(Y, k), \widehat{W}_{n}^{(j)}(Y, k), \tilde{P}_{n}^{(j)}(k), \widehat{F}_{n}(k)\right\}$ is defined by

$$
\begin{aligned}
& \left\{U_{j}, V_{j}, W_{j}, P_{j}(x, z), \tilde{F}(x, z)\right\} \\
& \quad=\sum_{n=-\infty}^{n=\infty} \int_{-\infty}^{\infty}\left\{\hat{U}_{n}^{(j)}(Y, k), \widehat{V}_{n}^{(j)}(Y, k), \widehat{W}_{n}^{(j)}(Y, k), \tilde{P}_{n}^{(j)}(k), \widehat{F}_{n}(k)\right\} \mathrm{e}^{\mathrm{i}((n z / l)+k x)} \mathrm{d} k,
\end{aligned}
$$

and it follows from the linearized boundary-layer equations (5.2)-(5.4) that

$$
\begin{gathered}
\mathrm{i} k \widehat{U}_{n}^{(j)}+(\mathrm{i} k \lambda)^{1 / 3} \frac{\mathrm{d} \widehat{V}_{n}^{(j)}}{\mathrm{d} \bar{\eta}}+\mathrm{i}\left(\frac{n}{l}\right) \widehat{W}_{n}^{(j)}=0, \\
\bar{\eta} \widehat{U}_{n}^{(j)}+\frac{(\mathrm{i} k \lambda)^{1 / 3}}{\mathrm{i} k} \widehat{V}_{n}^{(j)}+\frac{\mathrm{i} k}{(\mathrm{i} k \lambda)^{2 / 3}} \tilde{P}_{n}^{(j)}-\frac{\mathrm{d}^{2} \widehat{U}_{n}^{(j)}}{\mathrm{d} \bar{\eta}^{2}}=\widehat{F}_{n}^{(j)}(\bar{\eta}), \\
\bar{\eta} \hat{W}_{n}^{(j)}+\frac{\mathrm{i}(n / l)}{(\mathrm{i} k \lambda)^{2 / 3}} \tilde{P}_{n}^{(j)}-\frac{\mathrm{d}^{2} \widehat{W}_{n}^{(j)}}{\mathrm{d} \bar{\eta}^{2}}=\widehat{G}_{n}^{(j)}(\bar{\eta}),
\end{gathered}
$$

for $j=1,2$, where

$$
\begin{aligned}
& \bar{\eta} \equiv(\mathrm{i} k \lambda)^{1 / 3} Y \\
& \hat{F}_{n}^{(1)}=0 \\
(\mathrm{i} k \lambda)^{2 / 3} \hat{F}_{n}^{(2)}(\bar{\eta})=- & -\sum_{p=-\infty}^{p=\infty}\left[\hat{U}_{p}^{(1)}(Y, k) * \mathrm{i} k \hat{U}_{n-p}^{(1)}(Y, k)+\hat{V}_{p}^{(1)}(Y, k) * \frac{\partial}{\partial Y} \hat{U}_{n-p}^{(1)}(Y, k)\right. \\
& \left.+\mathrm{i}\left(\frac{n-p}{l}\right) \hat{W}_{p}^{(1)}(Y, k) * \widehat{U}_{n-p}^{(1)}(Y, k)\right]
\end{aligned}
$$

(not to be confused with the Fourier coefficient $\widehat{F}_{n}(k)$ of the roughness shape function $\tilde{F}(x, z)$ defined in $(5.10)$, see $(2.1))$

$$
\widehat{G}_{n}^{(1)}=0,
$$

and

$$
\begin{aligned}
(\mathrm{i} k \lambda)^{2 / 3} \widehat{G}_{n}^{(2)}(\bar{\eta})=\sum_{p=-\infty}^{p=\infty}\left[\hat{U}_{p}^{(1)}(Y, k) * \mathrm{i} k \widehat{W}_{n-p}^{(1)}(Y, k)+\widehat{V}_{p}^{(1)}(Y, k) * \frac{\partial}{\partial Y} \widehat{W}_{n-p}^{(1)}(Y, k)\right. \\
\left.+\mathrm{i}\left(\frac{n-p}{l}\right) \widehat{W}_{p}^{(1)}(Y, k) * \widehat{W}_{n-p}^{(1)}(Y, k)\right],
\end{aligned}
$$

where

$$
f(Y, k) * g(Y, k) \equiv \int_{-\infty}^{\infty} f\left(Y, k_{1}\right) g\left(Y, k-k_{1}\right) \mathrm{d} k_{1}
$$


denotes the convolution of the functions $f$ and $g$. The final results depend on the numerical coefficient of $1 / Y$ in the $\widehat{W}_{n}^{(2)}$ boundary condition (5.9) but not in the one in the $\hat{W}_{n}^{(1)}$ boundary condition (5.8). It is important to note that

$$
\widehat{F}_{n}^{(j)}(0)=\widehat{G}_{n}^{(j)}(0)=0 \text {. }
$$

The formula for the $n$th spanwise harmonic of the Fourier transformed first-order wall pressure $\tilde{P}_{n}^{(1)}$ is given in GSDC, while Appendix A shows that the $n$th spanwise harmonic of the Fourier transformed second-order wall pressure $\tilde{P}_{n}^{(2)}$ is given by

$$
\begin{aligned}
\tilde{P}_{n}^{(2)}= & -\frac{3 \pi(\mathrm{i} k \lambda)^{5 / 3}}{\left[k^{2}+(n / l)^{2}\right] \lambda 3^{1 / 3} \Gamma(1 / 3)} \int_{0}^{\infty}\left\{\left[I_{B}(t)+1 / \sqrt{3}\right] \mathrm{Ai}(t)\right. \\
& \left.-\left[I_{A}(t)-1 / 3\right] \operatorname{Bi}(t)\right\} \frac{\mathrm{d}\left[\hat{F}_{n}^{(2)}(t)+(n / k l) \hat{G}_{n}^{(2)}(t)\right]}{\mathrm{d} t} \mathrm{~d} t \\
= & -\frac{3 \pi(\mathrm{i} k \lambda)^{5 / 3}}{\left[k^{2}+(n / l)^{2}\right] \lambda 3^{1 / 3} \Gamma(1 / 3)} \int_{0}^{\infty}\left[\mathrm{Gi}(t)+\operatorname{Ai}(t) / 3^{1 / 2}\right] \frac{\mathrm{d}\left[\hat{F}_{n}^{(2)}(t)+(n / k l) \hat{G}_{n}^{(2)}(t)\right]}{\mathrm{d} t} \mathrm{~d} t \\
= & \frac{3 \pi(\mathrm{i} k \lambda)^{5 / 3}}{\left[k^{2}+(n / l)^{2}\right] \lambda 3^{1 / 3} \Gamma(1 / 3)} \int_{0}^{\infty}\left[\mathrm{Gi}^{\prime}(t)+\mathrm{Ai}^{\prime}(t) / 3^{1 / 2}\right]\left[\hat{F}_{n}^{(2)}(t)+(n / k l) \hat{G}_{n}^{(2)}(t)\right] \mathrm{d} t .
\end{aligned}
$$

Our interest is in the asymptotic behaviour of the second-order wall pressure as $x \rightarrow \infty$. Equations (4.1) and (4.2) show that this quantity is determined by the asymptotic behaviour of $\tilde{P}_{n}^{(2)}$ as $k \rightarrow 0$, and Appendix $\mathrm{B}$ shows that this latter quantity is given by

$$
\begin{aligned}
\tilde{P}_{n}^{(2)} \rightarrow & -\frac{3 \pi}{\left[k^{2}+(n / l)^{2}\right] \lambda 3^{1 / 3} \Gamma(1 / 3)} \sum_{p=-\infty}^{p=\infty} \int_{-\infty}^{\infty} \mathrm{d} k_{1} \int_{0}^{\infty}\left[\mathrm{Gi}^{\prime}(\bar{\eta})+\mathrm{Ai}^{\prime}(\bar{\eta}) / 3^{1 / 2}\right] \\
& \times\left[\widehat{U}_{n-p}^{(1)}\left((1-\kappa)^{1 / 3} \bar{\eta}, k-k_{1}\right) \mathrm{i} k_{1}+\widehat{V}_{n-p}^{(1)}\left((1-\kappa)^{1 / 3} \bar{\eta}, k-k_{1}\right)(\mathrm{i} k \lambda)^{1 / 3} \frac{\partial}{\partial \bar{\eta}}\right. \\
& \left.+\widehat{W}_{n-p}^{(1)}\left((1-\kappa)^{1 / 3} \bar{\eta}, k-k_{1}\right) \mathrm{i}(p / l)\right] \widehat{E}_{p}^{n}\left(\kappa^{1 / 3} \bar{\eta}, k_{1}\right) \mathrm{d} \bar{\eta} \\
\rightarrow & \frac{9 n k^{2 / 3} \lambda^{2} \ln k^{1 / 3}}{\left[k^{2}+(n / l)^{2}\right][\Gamma(1 / 3)]^{3}} \sum_{p=-\infty}^{p=\infty} \int_{-\infty}^{\infty} \frac{p \widehat{F}_{p}\left(k_{1}\right) \hat{F}_{n-p}\left(-k_{1}\right) k_{1}^{2 / 3}\left(-k_{1}\right)^{2 / 3}}{p^{2}+\left(l k_{1}\right)^{2}} \\
& \times\left[\frac{n(n-p)}{(n-p)^{2}+k_{1}^{2} l^{2}}-1\right] \mathrm{d} k_{1}, \quad \text { as } k \rightarrow 0 .
\end{aligned}
$$

\subsection{Behavior for $x \gg 1$}

It now follows from (3.6), (4.1)-(4.3) and (5.22) that (see (5.1) and (5.10))

$$
P(x, z)-\bar{P}(x)=h \hat{p}_{1}(0, z) x^{-8 / 3}+h^{2}\left[\hat{p}_{2}(0, z) x^{-5 / 3} \ln x^{1 / 3}+O\left(x^{-5 / 3}\right)\right],
$$

where

$$
\hat{p}_{2}(0, z) \equiv-\frac{2}{3^{1 / 2}} \Gamma(2 / 3) \sum_{\substack{n=-\infty \\ n \neq 0}}^{n=\infty} \tilde{a}_{n}^{(2)} \mathrm{e}^{\mathrm{i} n z / l} .
$$


with

$$
\begin{aligned}
\tilde{a}_{n}^{(2)}=\frac{-9(l \lambda)^{2} \mathrm{e}^{-\mathrm{i} \pi / 3}}{n[\Gamma(1 / 3)]^{3}} \sum_{p=-\infty}^{p=\infty} \int_{-\infty}^{\infty} \frac{p \hat{F}_{p}\left(k_{1}\right) \hat{F}_{n-p}\left(-k_{1}\right) k_{1}^{2 / 3}\left(-k_{1}\right)^{2 / 3}}{\left[p^{2}+\left(l k_{1}\right)^{2}\right]} \\
\times\left[\frac{n(n-p)}{(n-p)^{2}+\left(l k_{1}\right)^{2}}-1\right] \mathrm{d} k_{1} .
\end{aligned}
$$

Equation (5.23) fixes the arbitrary constants $\alpha$ and $\beta$ in (4.18)-(4.20) and shows that the first- and second-order contributions to spanwise variable wall pressure behave like $x^{-8 / 3}$ and $\left(\ln x^{1 / 3}\right) x^{-5 / 3}$, respectively. Equation (4.5) shows that the spanwise variable streamwise velocity grows like

$$
\begin{aligned}
u_{0}(x, y, z)-\bar{u}_{0}(x, y) \sim 3^{3 / 2} h^{2} \Gamma(2 / 3) x^{1 / 3} \ln x^{1 / 3} \frac{U_{B}^{\prime}(y)}{U_{B}^{2}(y)} & \\
& \times \sum_{\substack{n=-\infty \\
n \neq 0}}^{n=\infty} \pi_{n}^{\prime}(y, 0) \tilde{a}_{n}^{(2)} \mathrm{e}^{\mathrm{i} n z / l}, \quad \text { as } x \rightarrow \infty
\end{aligned}
$$

in the main boundary layer, while (4.19), (4.23), (4.30) and (4.31) show that the streamwise component of the wall-layer velocity perturbation behaves like

$$
U-\bar{U} \sim h^{2}\left[\ln x^{1 / 3} \tilde{U}_{2}(\eta, z)+O(1)\right], \quad \text { as } x \rightarrow \infty,
$$

where

$$
\begin{aligned}
\tilde{U}_{2}= & \frac{3}{2 \lambda^{2 / 3}} \frac{\partial^{2} \hat{p}_{2}(0, z)}{\partial z^{2}}\left[\hat{U}(\eta)+\hat{\mathrm{C}}_{H} \int_{0}^{\eta} \exp \left(-\frac{\eta^{3}}{9}\right) \mathrm{d} \eta\right. \\
& \left.+\frac{[\Gamma(1 / 3)]^{2} 3^{1 / 2}}{2 \pi 3^{1 / 3}} \int_{0}^{\eta} \mathrm{e}^{-\eta^{3} / 9} U\left(1 / 3,2 / 3, \eta^{3} / 9\right) \mathrm{d} \eta\right], \\
\hat{U}(\eta)= & \int_{0}^{\eta} \exp \left(-\bar{\eta}^{3} / 9\right) \int_{0}^{\bar{\eta}} \exp \left(\tilde{\eta}^{3} / 9\right) \mathrm{d} \tilde{\eta} \mathrm{d} \bar{\eta}
\end{aligned}
$$

with $\hat{C}_{H}$ chosen so that (4.29) is satisfied.

Since the spanwise average of the second-order wall pressure, $\tilde{P}_{0}^{(2)}$, is equal to zero, the linear and nonlinear components of the spanwise mean surface pressure exhibit the same $x^{-2 / 3}$ decay rate and the linear and nonlinear components of the spanwise average of the streamwise wall velocity exhibit the same $x^{-1}$ decay rate (see Appendix A of GSDC).

\section{Results and discussion}

Section 4 contains a theoretical description of the wakes at large downstream distances (in comparison to the scale of the roughness) behind a periodic array of roughness elements with a fundamental spanwise spacing of the order of the boundarylayer thickness. GSDC showed that this flow is eventually governed by linear equations and noted that these equations have an exact similarity solution when the spanwise variable component of the wall pressure decays as $x^{-\alpha}$, where $x$ denotes the scaled streamwise distance behind the roughness elements. However, they also noted that this same set of equations has an asymptotic solution corresponding to the more general algebraic/transcendental decay in wall pressure, of the form $x^{-\alpha} \ln x$. Their exact analytical solution for the strictly linear problem (where the scaled roughness height 
$h \ll 1)$ exhibited purely algebraic decay with the decay rate exponent, $\alpha$, being equal to $8 / 3$, while their numerical solutions for the full nonlinear case seemed to be consistent with a slower, $x^{-5 / 3}$ algebraic decay. GSDC's small $h$ analytical solution was extended to second order (i.e. to $O\left(h^{2}\right)$ ) in $\S 5$ of the present paper, where it was shown that the spanwise variable wall pressure actually behaves algebraically/transcendentally and decays at the slower $x^{-5 / 3} \ln x$ rate (see (5.23)) and, even more importantly, that the corresponding wall velocity undergoes a transcendental growth (see (5.27) and (6.5) below), accompanied by a progressively outward movement in the wallnormal location of the peak perturbation. The present section compares the weakly nonlinear, analytical solution with the $h=O(1)$ numerical solutions and confirms that the numerical solutions exhibit the same large $x$ asymptotic behaviour as the analytical solution and, therefore, that the weakly nonlinear asymptotic results of $\S 5.2$ have a broader relevance than their expected domain of applicability.

All of the computations are based on an array of roughness elements with the smooth shape function

$$
\tilde{F}(x, z)=\exp \left\{-\left[x^{2}+(z-\pi l)^{2}\right] / d^{2}\right\},
$$

for which the Fourier coefficient $\widehat{F}_{n}(k)$ is given by

$$
\widehat{F}_{n}(k)=\widehat{F}_{n}(0) \exp \left[-(k d / 2)^{2}\right]
$$

where

$$
\begin{gathered}
\hat{F}_{n}(0)=\frac{l D^{2}}{\pi} \mathrm{e}^{-(n D)^{2}-\mathrm{i} n \pi} \operatorname{Re} \operatorname{erf}(\pi / 2 D+\mathrm{i} n D), \\
D \equiv d / 2 l .
\end{gathered}
$$

Here Re denotes the real part of a complex quantity and erf denotes the error function. The numerical procedure for solving the nonlinear wall-layer problem (3.19)-(3.24) (which can also be used for the analogous two-dimensional problem of Smith $(1976 a, b))$ is described in GSDC.

\subsection{Solutions in the vicinity of the roughness array}

Figure 2, which is a plot of the wall-shear stress distribution along the symmetry plane $z=\pi l$ of the roughness element, gives some indication of the local flow field for $O(1)$ values of $h$. It indicates that there is a weak retardation of the near-wall flow ahead of the roughness elements, followed by a strong acceleration that leads to a prominent peak in centreline wall shear just ahead of $x=0$ (i.e. streamwise location of maximum roughness height). The flow rapidly decelerates over the rear half of the roughness element until the wall shear reaches a minimum between $x \approx 5$ and $x \approx 10$ (i.e. $x / d \approx 0.725$ to $x / d \approx 1.45$ ) and begins a recovery to its unperturbed state to the leading order in the high-Reynolds-number expansion (3.16). The minimum wall shear decreases with increasing $h$, but remains positive for all values of roughness height as shown in figure 2. The flow behind the roughness element appears to be on the verge of separation at $\lambda^{1 / 3} h=5$, but there is little likelihood of a spontaneous onset of unsteady vortex shedding within the wake region (as observed for sufficiently large values of the roughness height by Acarlar \& Smith (1987) and Klebanof, Cleveland \& Tidstrom (1992)), since the flow remains fully attached throughout the range $\lambda^{1 / 3} h \leqslant 5$. The stationary solutions shown in figure 2 are, therefore, realizable, in principle.

GSDC found that the numerically computed spanwise variable component of the wall pressure was in good agreement with the linear analytical result up to $x=10^{3}$ 


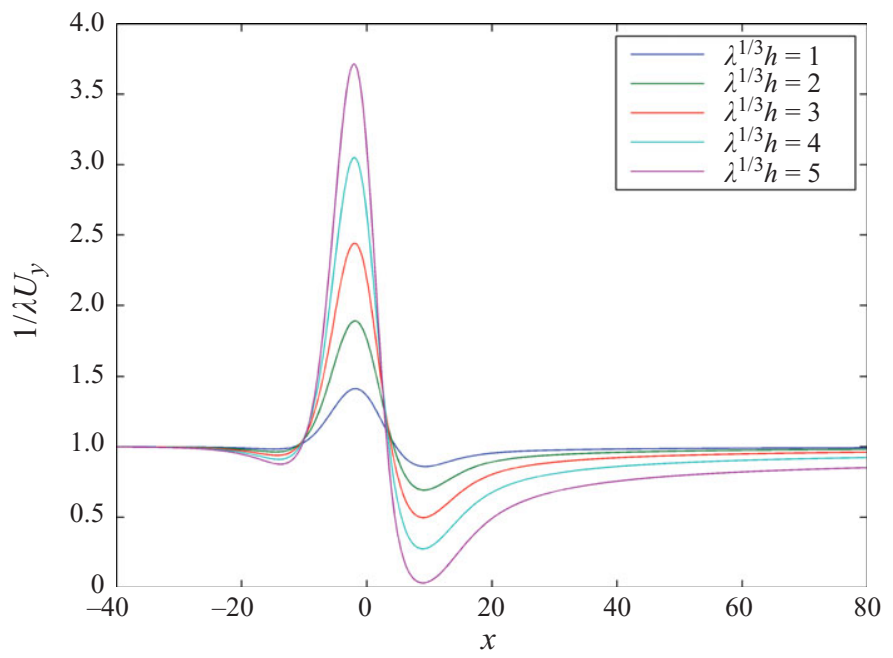

FIGURE 2. Scaled wall shear distribution along the symmetry plane $z=\pi l$ of the roughness element.

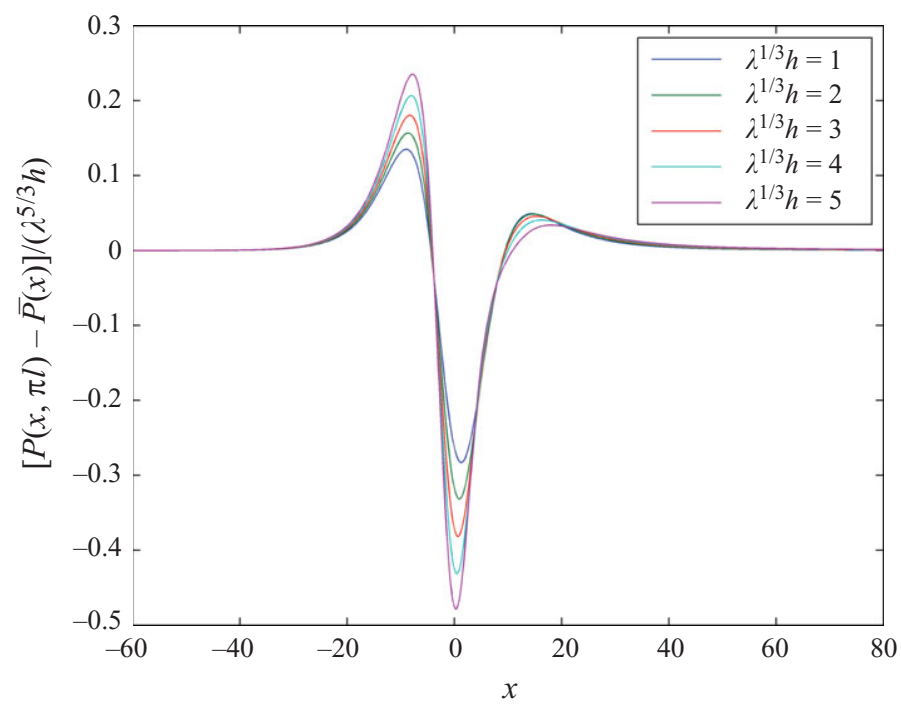

FIGURE 3. Normalized spanwise variable component of surface pressure distribution over the roughness elements for $l=5, d=6.9$ computed from the nonlinear solution.

when the roughness height $h$ was relatively small - of the order of 0.1 or so - and that the agreement between the nonlinear and linear results even extended up to $h=1$ or so if the comparison was restricted to the immediate vicinity of the roughness array. Figure 3 displays the numerically computed spanwise variable component of the surface pressure normalized by $h \lambda^{5 / 3}$. It is similar to figure 10 of GSDC, but carries the computations to much higher $h$ values (the symbol $h$ should be replaced by $\lambda^{1 / 3} h$ in the caption of that figure). The results show the increasing effects of nonlinearity as $h$ becomes large, especially along the peaks and valleys of the streamwise pressure distribution. 


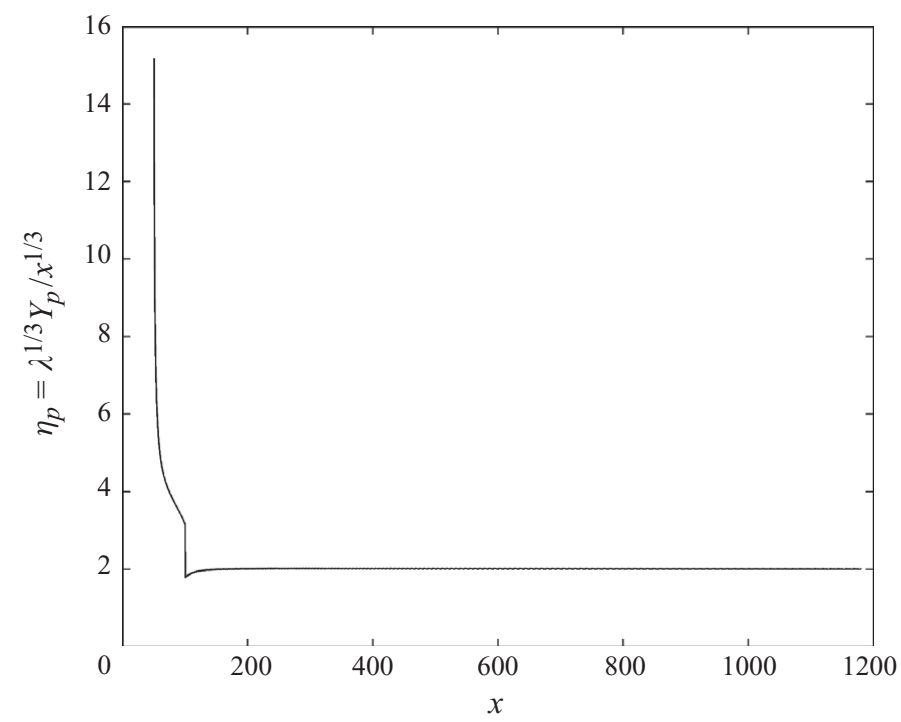

FIGURE 4. Similarity coordinate of peak velocity defect calculated from numerical solution for $\lambda^{1 / 3} h=2$ at the spanwise location of maximum velocity defect.

\subsection{Downstream behaviour for $x \gg 1$}

We have verified that the numerical solutions can be treated as a small perturbation about the linear profile $\lambda Y$ as $x$ becomes large and, therefore, that they satisfy the linear equations (4.9)-(4.11). The linearity of the wake perturbations was also demonstrated by the direct numerical simulation solutions of Fischer \& Choudhari (2004). The asymptotic solution (4.18)-(4.20) to (4.9)-(4.11) with $\alpha=5 / 3$ implies that the peak wake velocity will occur at a constant value of $\eta$, say $\eta=\eta_{p} \equiv \lambda^{2 / 3} Y_{p} / x^{1 / 3}$, and will remain constant when scaled with $\ln x^{1 / 3}$, i.e. that the ratio $(U-\bar{U})\left(\eta_{p}, z\right) / \ln x^{1 / 3}$ will remain constant as $x \rightarrow \infty$. These asymptotic predictions are consistent with the appropriately scaled numerical solution for $\lambda^{1 / 3} h=2$, as seen from the nearly flat large $x$ asymptote in figure 4 (which is a plot of $\eta_{p}$ at the spanwise location of the maximum velocity defect) and, somewhat less convincingly, in figure 5. The latter result could be improved by extending the grid further downstream, but the additional computational effort does not seem to be warranted. The discontinuity in figure 4 is due to the occurrence of a double peak.

Figure 6 is a plot of the scaled spanwise variable surface pressure at large downstream distances. The black curve is computed from the lowest-order contribution to the $O\left(h^{2}\right)$ term in the asymptotic solution (5.23). The coloured curves, which are calculated from the numerical solution, show that the overall level and asymptotic decay rate of the analytical result are in remarkably good agreement with the numerical computations - with the decay rates and overall level being virtually identical when $\lambda^{1 / 3} h \geqslant 3$, which is consistent with the slightly faster than $h^{2}$ increase in the overall level of the numerical results when $\lambda^{1 / 3} h<3$. This suggests that the numerically computed pressures have not fully achieved the slower $x^{-5 / 3} \ln x$ decay for $\lambda^{1 / 3} h$ less than about 3 . This can either be attributed to $O\left(x^{-5 / 3}\right)$ contribution to the $O\left(h^{2}\right)$ term in the weakly nonlinear solution (5.23) or to the neglect of the faster decaying $O(h)$ linear term. However, we do not pursue this because of the significant memory requirements needed for the nonlinear computations at large values of $x$. 


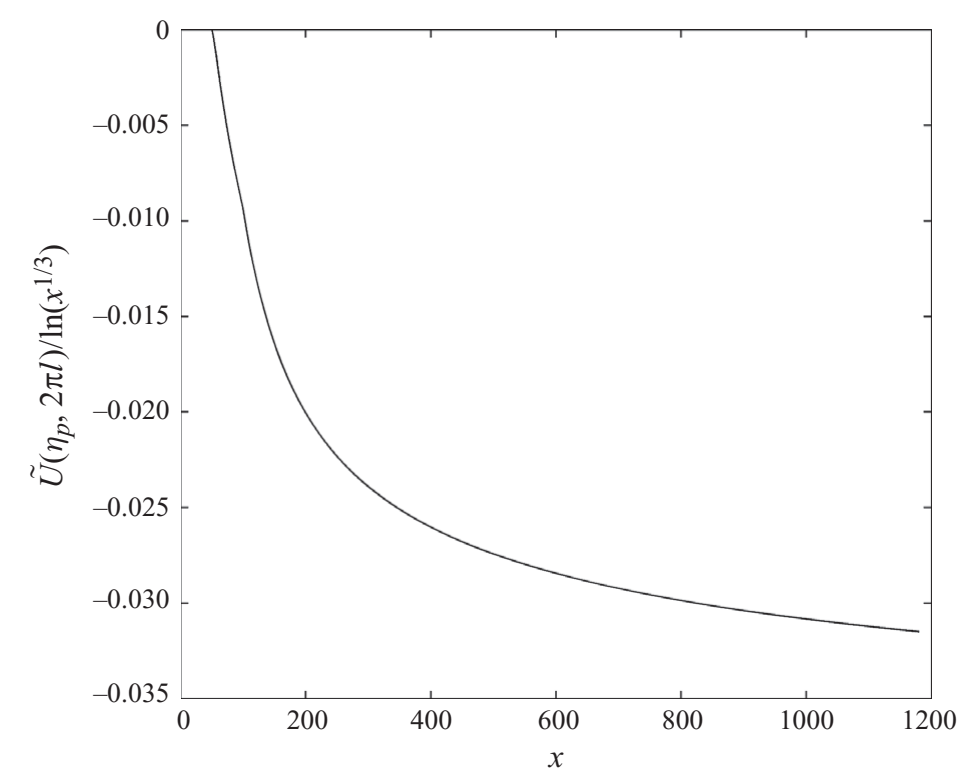

FigURE 5. Scaled peak velocity defect calculated from numerical solution for $\lambda^{1 / 3} h=2$.

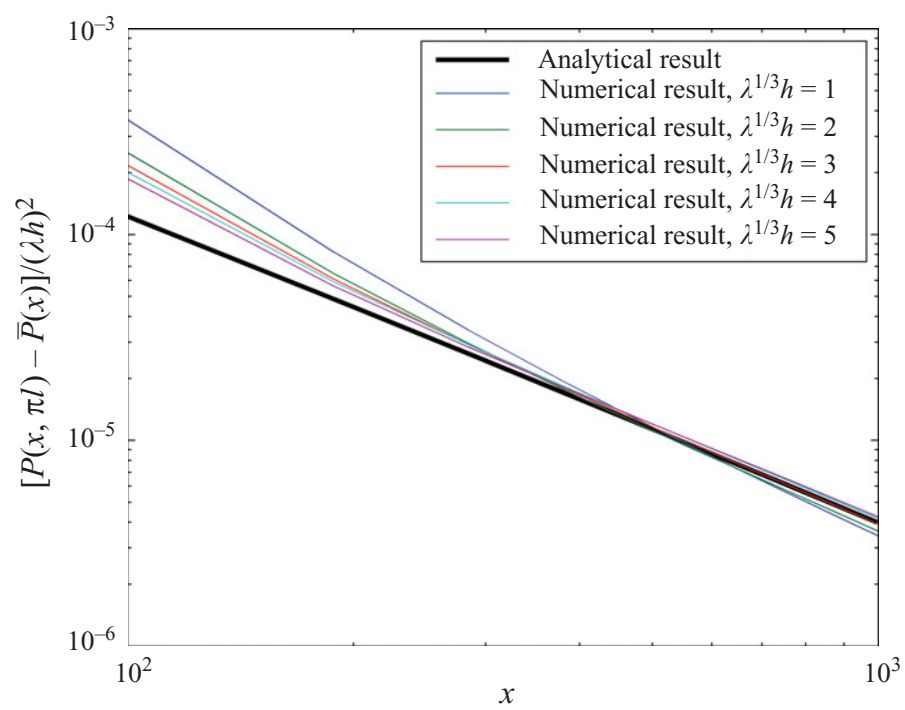

FiguRE 6. Normalized spanwise variable surface pressure decay rates for $l=5, d=6.9$.

Since the curves show that the numerically computed wall pressure is proportional to $h^{2}$ for $\lambda^{1 / 3} h \geqslant 3,(5.26)$ and (5.27) imply that the streamwise wake velocity is also roughly proportional to $h^{2}$ and, therefore, to $R e_{k}$, where $R e_{k}$ denotes the Reynolds number based on roughness height and the boundary-layer velocity at this height. This, in turn, implies that the streamwise wake energy should scale with $\left(\operatorname{Re}_{k}\right)^{2}$ as found experimentally by White \& Ergin (2003) and in numerical computations by Choudhari \& Fischer (2005). The present weakly nonlinear theory, therefore, provides a plausible explanation for those findings. 
GSDC found that the peak value of the streamwise component of the spanwise variable wall velocity decays as $1 / x$ in the downstream wall layer in the strictly linear case. But the present result shows that it actually increases like $\ln x$ as $x \rightarrow \infty$ in the fully nonlinear case. This does not, however, destroy the linearity of the downstream flow because the peak velocity moves upward into the main boundary layer like $x^{1 / 3}$ as $x \rightarrow \infty$, which means the local Blasius velocity (about which the equations are linearized) also increases like $x^{1 / 3}$ and, therefore, always remains larger than the wake velocity perturbation.

This increase in the spanwise variable component of the streamwise velocity perturbation, which is generated by the interaction between the various spanwise Fourier modes in the nonlinear case, produces an effect that is very similar to the well-known (Landahl 1980) 'lift-up' effect that is generated by a purely linear mechanism and is probably responsible for the transient growth that occurs in the purely linear case considered in GSDC. The former (modal interaction) mechanism cannot occur in the strictly linear case because of the absence of these interactions in that case. However, both the $O(h)$ and $O\left(h^{2}\right)$ streamwise velocity components of the corresponding two-dimensional problem decay at the same rate, which reinforces the notion that the streamwise velocity amplification is associated with the spanwise modal interactions - since these interactions cannot occur in the two-dimensional case.

It might, at first glance, appear that the asymptotic small $h$ expansion should break down when the $O\left(h^{2}\right)$ term becomes larger than the $O(h)$ result. But there is no reason why an asymptotic solution cannot remain valid when one or two of its terms are smaller than the remaining higher-order terms in some region of space. A simple example of this behaviour is given by a classical linear acoustics problem of calculating the acoustic radiation from a compact source with a net mass flux (at any instant of time) of the order of the compactness parameter (i.e. the source size relative to acoustic wavelength scale). When the acoustic field is expanded in powers of this small parameter, the monopole contribution is excluded from the lowest-order term in this multipole expansion (which would correspond to linear solution in the present case) but not from the higher-order terms (which would correspond to nonlinear solution). Since the weak monopole decays more slowly than the lowest-order solution (which is of higher multipole order), the acoustic far-field will be dominated by the higher order terms, but the expansion will remain valid (with some rearrangement of the terms with varying $x$ ). It may be helpful to think about this as subtracting out the linear solution and constructing an asymptotic expansion of the remainder.

The good agreement with the $O(1)$ numerical solution suggests that all of the higher order terms have the same decay rate and, therefore, that the expansion beyond the $O(h)$ term proceeds in the expected manner. The close agreement at a relatively large value of the 'small parameter' $h$ is also not that unusual. There are many examples of this in chapters 3 and 4 of the classical text by Bender \& Orszag (1978).

Figure 7 compares the spanwise variation in the asymptotic wall pressure determined from the $\lambda^{1 / 3} h=3, d=6.9, l=5$ numerical computation with the $O\left(h^{2}\right)$ analytical result. Both solutions are roughly sinusoidal, but the numerical result is slightly concave on the centreline $z=\pi l$ of the roughness elements, which is important because (5.27) and (5.28) show that the streamwise wall velocity is proportional to the second derivative of the wall pressure.

The good agreement with the numerical results suggests that the relatively simple analytical result (5.26)-(5.29) can be used to study the effects of various roughness shapes and parameters even when the nonlinearity is fairly strong. The wall pressure 


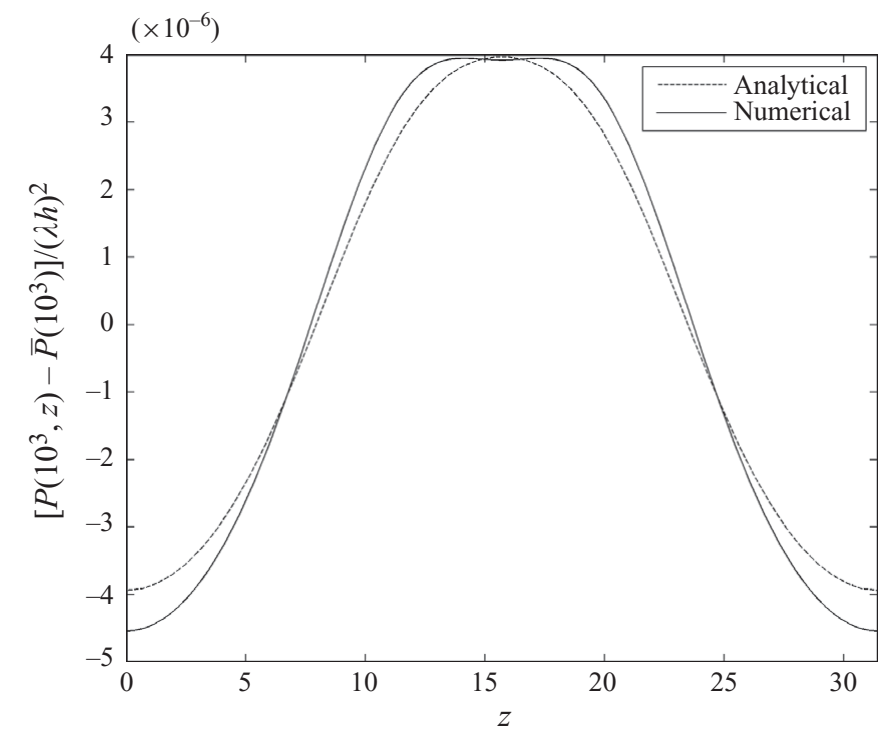

FIGURE 7. Comparison of spanwise variation in scaled wall pressure computed from the $\lambda^{1 / 3} h=3$ numerical solution (for $d=6.9, l=5$ ) with the $O\left(h^{2}\right)$ analytical result.

is of particular importance because (5.23), (5.27) and (5.28) show that the spanwise variable streamwise velocity in the wall layer is directly proportional to the second spanwise derivative of this quantity and similar results apply to the other wall layer velocity components.

Figure 8 is a plot of the spanwise variation in the $O\left(h^{2}\right)$ asymptotic wall pressure calculated from the $O\left(h^{2}\right)$ term in (5.23) for various values of $d$ and $l$. Notice that the pressure peak lies directly behind the roughness element and that it increases in magnitude when the distance $l^{*}$ between the roughness elements increases relative to the local boundary-layer thickness, $\delta^{*}$. It reaches a maximum when $D / \pi=d / 2 \pi l$ is somewhere between 0.3 and 0.4 , i.e. when the roughness diameter is close to a third of the roughness spacing. Since the roughness elements are non-compact, their tails tend to overlap when $D / \pi \geqslant 0.6$, which is partially responsible for the $D / \pi=0.8$ curve being so flat. The dominant contribution to these results comes from the $n=1$ mode interacting with $p=1$ term and the $n=-1$ mode interacting with $p=-1$ term in the sum (5.25), which involves the $n-p=0$ spanwise uniform mode. So it follows from (5.27) and (5.28) that most of the nonlinear growth in the streamwise wall-layer velocity is caused by a 'linear' interaction between the fundamental mode and the two-dimensional distorted flow. This is somewhat analogous to what happens in the linear case considered in GSDC, where most of the algebraic growth results from the interaction between the fundamental harmonic and the two-dimensional flow that results from the slow growth of the boundary layer over the viscous length scale.

Figures 9 and 10 are plots of the uniformly valid (Van Dyke 1975, p. 96) multiplicative 'composite' wall-layer/main boundary-layer solution

$$
u(x, y, z) / \varepsilon h^{2}=-\ln x^{1 / 3} \tilde{U}_{2}(\eta, z) \frac{2 y \lambda \tilde{u}^{*}(y, z)}{9}\left[\frac{\partial^{2} \tilde{p}_{0}^{(0)}(0, z)}{\partial z^{2}}\right]^{-1}
$$


(a)

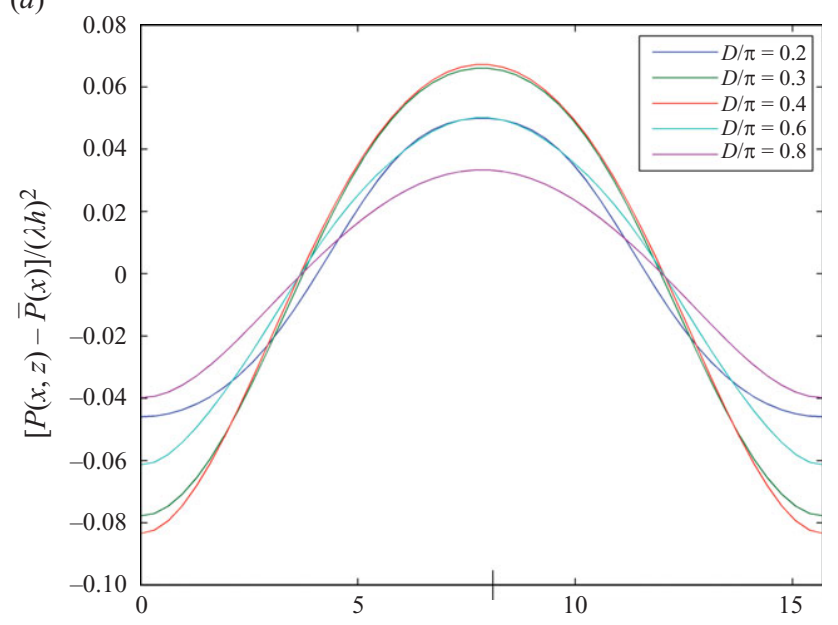

(b)

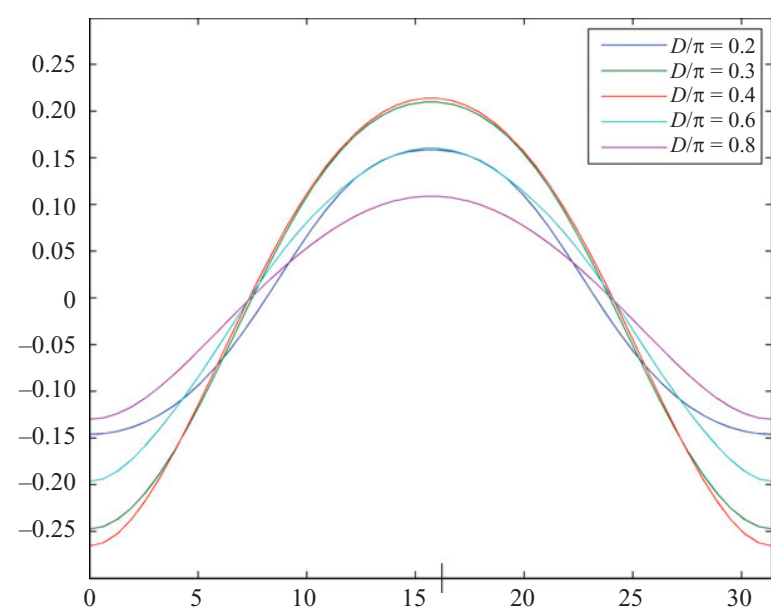

(c)

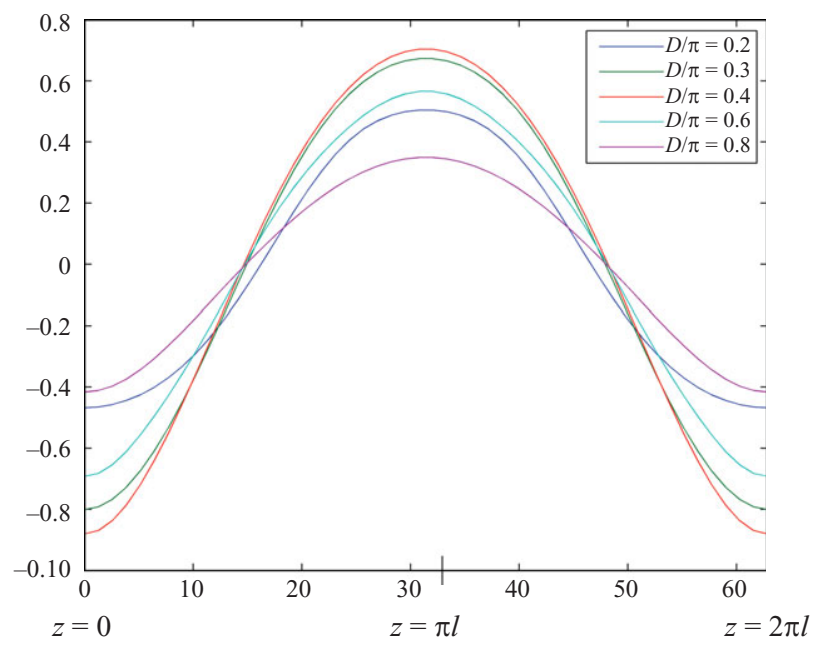

FiguRE 8. Spanwise variation in scaled asymptotic wall pressure calculated from the $O\left(h^{2}\right)$ asymptotic solution at $x=700$. (a) $l=2.5$. (b) $l=5$. (c) $l=10$. 
(a)

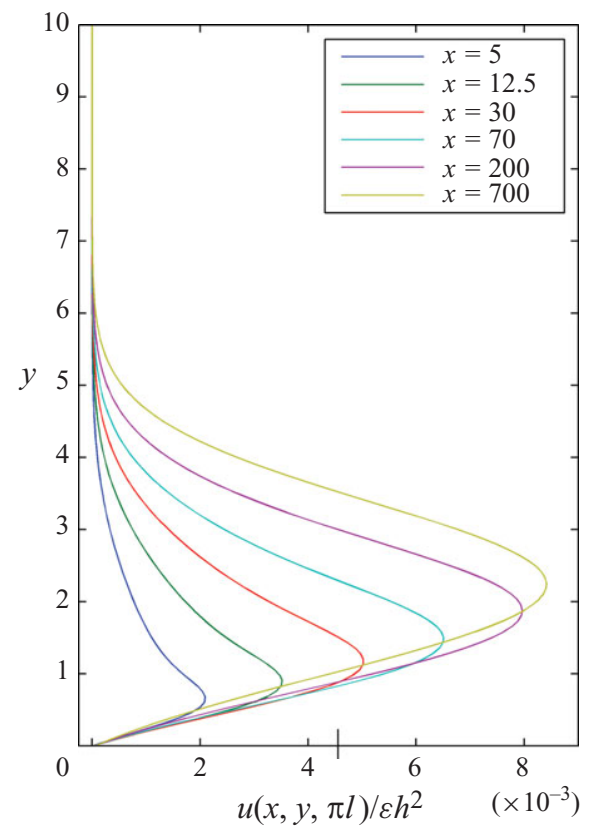

(b)

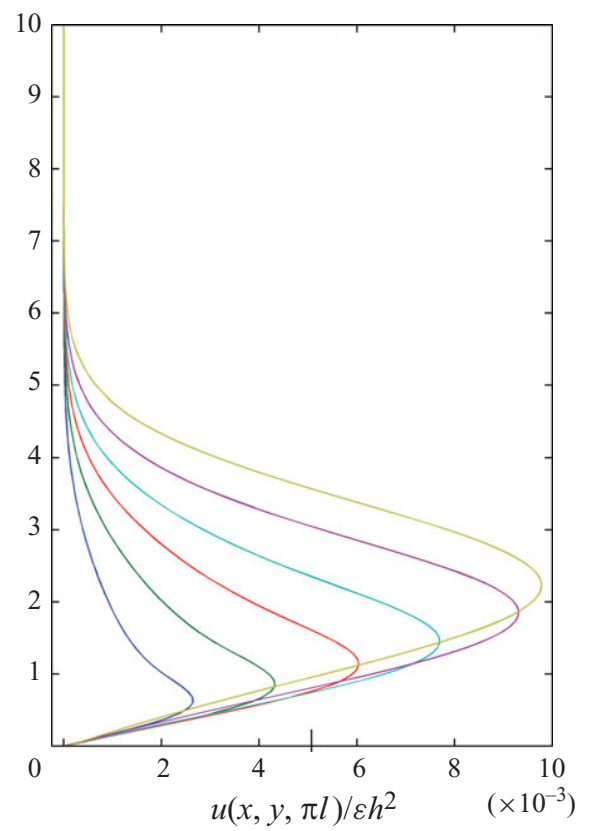

FIGURE 9. Effect of plan form diameter $d$ on scaled streamwise velocity perturbation at $z=\pi l$ : wall-normal profiles of $u / \varepsilon h^{2}$ calculated from uniformly valid $O\left(h^{2}\right)$ asymptotic solution at large values of $x$. (a) $d=6.9$ and $(b) d=13.8$ (array spacing $l=5$ in both cases).

(a)

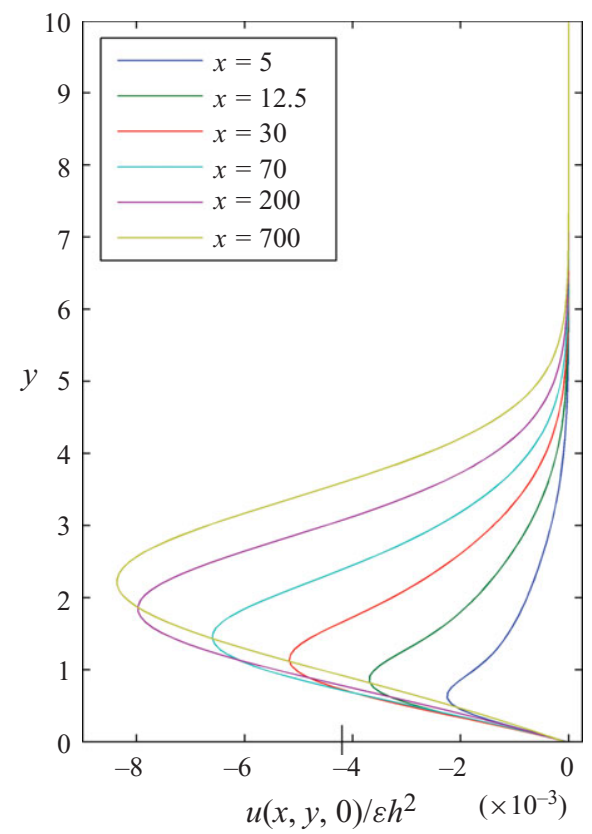

(b)

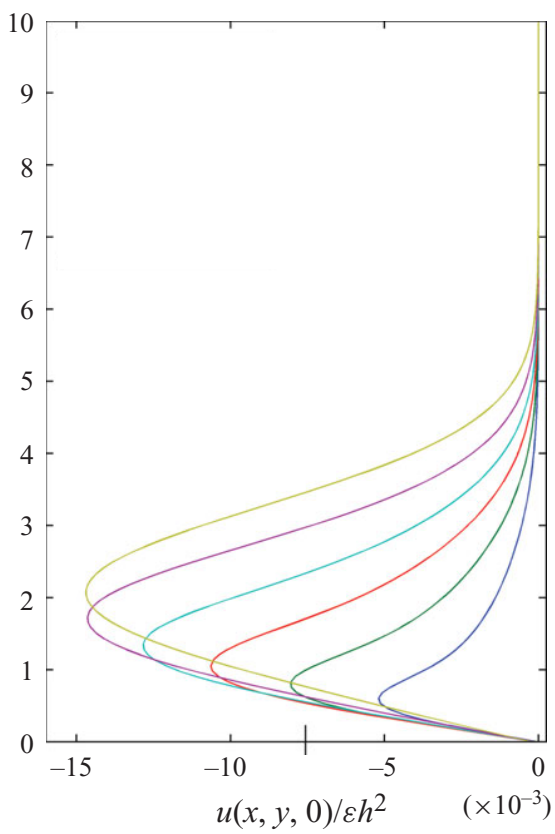

FIGURE 10. Same as figure 9, except $z=0$ instead of $z=\pi l$. 
formed from (5.26)-(5.29) with $\tilde{u}^{*}(y, z)$ given by

$$
\tilde{u}^{*}(y, z) \equiv 3^{3 / 2} \Gamma(2 / 3) \frac{U_{B}^{\prime}(y)}{U_{B}^{2}(y)} \sum_{\substack{n=-\infty \\ n \neq 0}}^{n=\infty} \pi_{n}^{\prime}(y, 0) \tilde{a}_{n}^{(2)} \mathrm{e}^{\mathrm{i} n z / l} .
$$

Perturbation profiles at the roughness centreline $z=\pi l$ are shown in figure 9 , whereas those in between the adjacent roughness elements $(z=0)$ are plotted in figure 10 . These figures show that the peak in spanwise variable streamwise velocity eventually moves up into the main boundary layer or main deck, where the Blasius velocity is more uniform and the perturbation increases at a much more rapid $x^{1 / 3} \ln x$ rate. But since the velocity maximum tends to remain in the wall layer this mainly shows up as an increase in the wall-normal wake thickness. This causes the total wake energy to exhibit algebraic/transcendental growth in the nonlinear case, while the GSDC paper shows that it decays like $x^{-2 / 3}$ in the strictly linear case. The positive value of the velocity in figure 9 suggests that the wake flow reversal shown in figures 6 to 9 of GSDC now takes place on the shorter inner scale $x$ of the roughness elements rather than on the longer outer scale $X$ on which the mean flow evolves. This is consistent with the algebraic growth occurring on the inner scale, $x$, in the nonlinear case and on the outer scale, $X$, in the strictly linear case.

\subsection{Transient growth on body length scale}

Equations (3.1) and (4.5) show that the nonlinear solution (both numerical and analytical) will become invalid when $\varepsilon^{2} x^{1 / 3} \ln x^{1 / 3}=O(1)$. But GSDC showed that the mean boundary-layer growth causes the expansion (3.1) to break down on the much shorter $X \equiv x^{*} / x_{0}^{*}=1+\delta x=O(1)$ length scale, and that the flow in the corresponding outer region is governed by the linearized boundary region (LBR) equations (Kemp 1951). The relevant boundary value problem is described in $\S 5$ of GSDC. The only difference is that the last curly bracket in the outer expansion (5.2) of GSDC is multiplied by the scale factor $\ln \delta^{-1 / 3}=-\ln \varepsilon$ in the present case. In other words, the only required modification is a slight change in the gauge functions in the outer expansion (5.2) of GSDC which now becomes

$$
\begin{aligned}
\{u, v, w, p\}= & \left\{U_{B}\left(\eta_{B}\right), \delta V_{B}\left(X, \eta_{B}\right), 0,0\right\}+\varepsilon^{3}\{\tilde{\tilde{u}}(X, y), \delta \overline{\tilde{v}}(X, y), 0, \overline{\tilde{p}}(X, y)\}+\cdots \\
& -\varepsilon^{3 \alpha-4} \ln \varepsilon\left\{\tilde{u}(X, y, z), \delta \tilde{v}(X, y, z), \delta \tilde{w}(X, y, z), \delta^{2} \tilde{p}(X, y, z)\right\} \\
& +O\left(\varepsilon^{3 \alpha-4}\right) \ldots,
\end{aligned}
$$

where

$$
V_{B}\left(\eta_{B}\right)=\frac{1}{2}\left(v^{*} U_{\infty} / x^{*}\right)^{1 / 2}\left[\eta_{B} F^{\prime}\left(\eta_{B}\right)-F\left(\eta_{B}\right)\right]
$$

and $\eta_{B} \equiv\left(y^{*} / x^{*}\right) \sqrt{x^{*} U_{\infty} / v^{*}}$ denotes the Blasius variable. So the scaled energy plotted in figure 13 of GSDC remains unchanged (the lower case $x$ should be a capital $X$ in the horizontal scale of that figure), which means that the primary consequence of the non-parallel flow effects is still a reduction in growth and eventual decay of the streamwise velocity. This differs significantly from the purely linear case considered in GSDC, where the algebraic growth in the wake velocity is produced by the non-parallel flow effects in the outer region.

We used the numerically computed wall pressure shown in figure 7 to calculate the upstream boundary conditions for the LBR equations that govern the flow on the long streamwise length scale $X$ for $\lambda^{1 / 3} h=3$. Figure 11 is a plot of the spanwise variable component of the scaled streamwise velocity half-way between the roughness elements for that value of $h$. It shows that the wakes (which were initially concentrated 


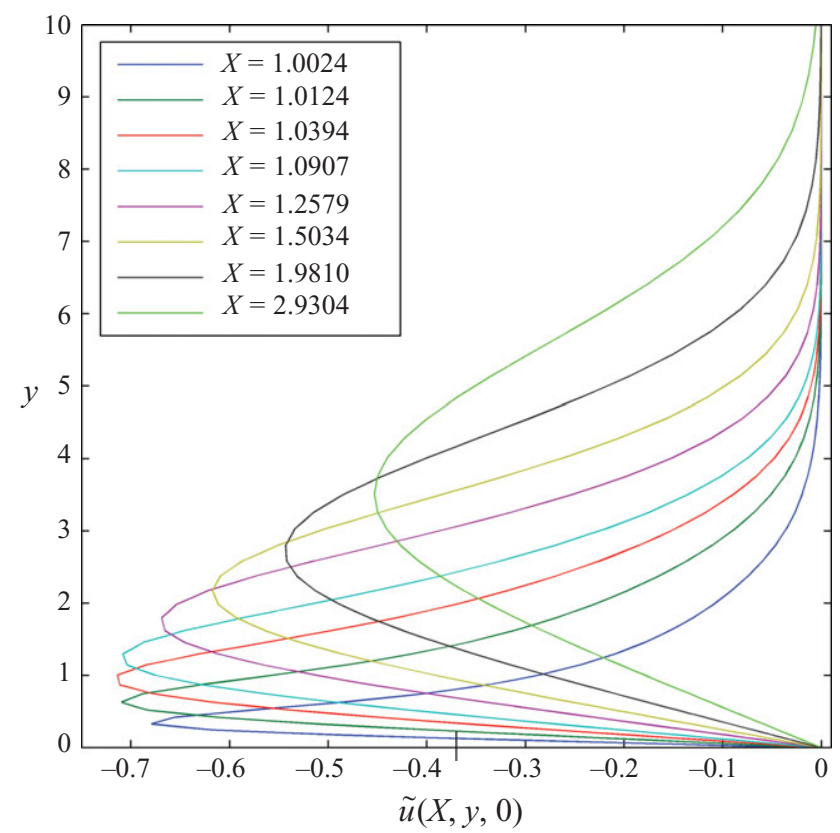

FIGURE 11. Scaled streamwise velocity perturbation $\tilde{u}$ as a function of $y$ at the spanwise location $z=0$ between the roughness elements for $\lambda^{1 / 3} h=3, l=5, d=6.9$.

in the wall layer in the intermediate region, $x \gg 1, X-1 \ll 1)$ continue to move out into the main boundary layer as they progress downstream but do not undergo the sign reversals exhibited by the wakes for the strictly linear cases shown in figures 6 and 7 of GSDC - presumably because this has already occurred in the inner region in this case. (The lower case $x \mathrm{~s}$ should be capital $X \mathrm{~s}$ in the legends of figures 6 to 9 of GSDC.)

Figure 11 also shows that the initial increases in the streamwise wake energy in figure 13 of GSDC are primarily due to wake spreading and not due to a decrease in the streamwise velocity minimum (see discussion for figures 9 and 10 above).

\section{Summary and concluding remarks}

This paper is based on a high-Reynolds-number asymptotic solution for the flow over a spanwise periodic array of relatively small roughness elements that was developed by GSDC. The roughness elements are assumed to be more or less circular in plan form with the plan form size and array spacing being of the order of the local boundary-layer thickness. The roughness height is assumed to be small enough to produce only local separation. The present work extends the small $h$ asymptotic results of GSDC to show that nonlinearity can have a profound effect on the evolution of the wake disturbances behind the roughness elements. It shows that the downstream wakes consist of streak-like perturbations that exhibit algebraic/transcendental growth and that the location of growth onset moves closer to the roughness elements with increasing $h$, even when the scaled roughness height $h$ is relatively modest - a trend that has previously been observed in direct numerical simulations for a non-smooth roughness shape.

Transient growth behaviour over long streamwise length scales of the order of the downstream distance from the leading edge was identified by Andersson et al. 
(1999), Luchini (2000) and Tumin \& Reshotko (2001) in the context of the linearized boundary region equations. However, the primary focus of these studies was on optimal growth characteristics which may not be achieved for realistic roughness configurations. The present paper examines realizable transient growth behaviour in the context of inverse, nonlinear boundary-layer equations with zero displacement and shows that the streak-like velocity perturbations can exhibit a weaker, i.e. suboptimal growth of the transcendental form $\ln x$ as $x \rightarrow \infty$ on the roughness scale. And most importantly, the theory is able to predict the quadratic dependence of the wake perturbation amplitudes on roughness height noted in previous experiments (White \& Ergin 2003) and numerical computations (Choudhari \& Fischer 2005). In addition to reproducing the dominant nonlinear receptivity characteristics, the theory provides a rational basis for the previously observed linear behaviour of the velocity distortions across the region of transient growth and subsequent decay.

The $O\left(h^{2}\right)$ asymptotic solution was found to be in good agreement with the numerical solution in the intermediate wake region where $x \gg 1$ and $0<X-1 \ll 1$ even for moderately large values of the roughness height parameter $h$-which means that the relatively simple analytical result (5.26)-(5.29) can be used to estimate the effects of various roughness shapes and parameters even when the nonlinearity is fairly strong. It is only necessary to evaluate the inverse Fourier transform $\widehat{F}_{n}(k)$ of the roughness-shape function $\tilde{F}(x, z)$, as was done in $\S 6$ of the present paper for the smooth Gaussian function (6.1) and in $\S 8$ of GSDC for the circular disk defined in (8.4). $\left(|z-\pi l|\right.$ should be replaced by $\sqrt{x^{2}+(z-\pi l)^{2}}$ in that equation.) Since the theoretical formulation is valid for arbitrary roughness configurations, the present computations can easily be extended to other roughness shapes and, in particular, to compact shapes such as circular disks, which are likely to result in flow separation at smaller values of roughness height than for the smoother Gaussian shape.

The authors gratefully acknowledge the support of the Aerothermodynamics element of NASA's subsonic fixed wing project and the Aerothermodynamics and Plasma Dynamics element of the hypersonics project. A.S. would like to thank Dr A. Afjeh, Professor and Chair, and Dr R. Hixon, Associate Professor, MIME Department, University of Toledo.

\section{Appendix A}

This appendix gives the details of the derivation of the formula (5.21) for the $n$th spanwise harmonic of the second-order wall pressure $\tilde{P}_{n}^{(2)}$. Eliminating $\widehat{V}_{p}^{(j)}$ between (5.11) and (5.12) yields

$$
\frac{\mathrm{d}^{3} \widehat{U}_{n}^{(j)}}{\mathrm{d} \bar{\eta}^{3}}-\bar{\eta} \frac{\mathrm{d} \widehat{U}_{n}^{(j)}}{\mathrm{d} \bar{\eta}}=-\left(\frac{n}{k l}\right) \widehat{W}_{n}^{(j)}-\frac{\mathrm{d} \hat{F}_{n}^{(j)}}{\mathrm{d} \bar{\eta}},
$$

where $\widehat{U}_{n}^{(j)}$ must satisfy the boundary condition

$$
\frac{\mathrm{d}^{2} \widehat{U}_{n}^{(j)}}{\mathrm{d} \bar{\eta}^{2}}=\frac{(\mathrm{i} k \lambda)^{1 / 3} \tilde{P}_{n}^{(j)}}{\lambda}-\widehat{F}_{n}^{(j)}(0), \quad \text { for } \bar{\eta}=0, j=1,2 .
$$


Equations (5.13) and (A 1) can be solved to obtain

$$
\begin{aligned}
\hat{W}_{n}^{(j)}=-\frac{\mathrm{i} n \pi \tilde{P}_{n}^{(j)}}{(\mathrm{i} k \lambda)^{2 / 3} l} & \operatorname{Gi}(\bar{\eta})+\widehat{\alpha}_{n}^{(j)} \operatorname{Ai}(\bar{\eta}) \\
& +\pi \int_{\bar{\eta}}^{\infty} \operatorname{Ai}(t) \operatorname{Bi}(\bar{\eta}) \widehat{G}_{n}^{(j)}(t) \mathrm{d} t+\pi \int_{0}^{\bar{\eta}} \operatorname{Ai}(\bar{\eta}) \operatorname{Bi}(t) \widehat{G}_{n}^{(j)}(t) \mathrm{d} t,
\end{aligned}
$$

and

$$
\begin{aligned}
& \hat{U}_{n}^{(j)}+(n / k l) \hat{W}_{n}^{(j)}=\beta_{n}^{(j)} I_{A}(\bar{\eta})+\pi \int_{\bar{\eta}}^{\infty} I_{B}(\bar{\eta}) \operatorname{Ai}(t) \frac{\mathrm{d}\left[\hat{F}_{n}^{(j)}(t)+(n / k l) \widehat{G}_{n}^{(j)}(t)\right]}{\mathrm{d} t} \mathrm{~d} t \\
& +\pi \int_{0}^{\bar{\eta}}\left\{\left[I_{A}(\bar{\eta})-I_{A}(t)\right] \operatorname{Bi}(t)+I_{B}(t) \operatorname{Ai}(t)\right\} \frac{\mathrm{d}\left[\hat{F}_{n}^{(j)}(t)+(n / k l) \widehat{G}_{n}^{(j)}(t)\right]}{\mathrm{d} t} \mathrm{~d} t, \quad
\end{aligned}
$$

for $j=1,2$ where

$$
I_{A}(\eta) \equiv \int_{0}^{\eta} \operatorname{Ai}(t) \mathrm{d} t, \quad I_{B}(\eta) \equiv \int_{0}^{\eta} \operatorname{Bi}(t) \mathrm{d} t
$$

the $\beta_{n}^{(j)}, \hat{\alpha}_{n}^{(j)}$ are constants to be determined by the boundary conditions and the Ai and Gi denote the Airy functions defined in Abramowitz \& Stegun (1965, p. 446 and 448). It now follows from (5.7), (A 3) and equations (10.4.4) and (10.4.42) of Abramowitz \& Stegun that

$$
\hat{\alpha}_{n}^{(j)}=\frac{\mathrm{i} n \pi \tilde{P}_{n}^{(j)}}{(\mathrm{i} k \lambda)^{2 / 3} l \sqrt{3}}-\pi \sqrt{3} \int_{0}^{\infty} \operatorname{Ai}(t) \widehat{G}_{n}^{(j)}(t) \mathrm{d} t
$$

for $j=1,2,3, \ldots$, while differentiating (A 4 ) with respect to $\eta$ and using the boundary condition (A 2) shows that

$$
\begin{aligned}
\widehat{U}_{n}^{(j)}= & -(n / k l) \widehat{W}_{n}^{(j)}+I_{A}(\bar{\eta})\left[k^{2}+(n / l)^{2}\right] \frac{\lambda 3^{1 / 3} \Gamma(1 / 3) \tilde{P}_{n}^{(j)}}{(\mathrm{i} k \lambda)^{5 / 3}} \\
& +\pi \int_{\bar{\eta}}^{\infty}\left[I_{B}(\bar{\eta})+3^{1 / 2} I_{A}(\bar{\eta})\right] \mathrm{Ai}(t) \frac{\mathrm{d}\left[\widehat{F}_{n}^{(j)}(t)+(n / k l) \widehat{G}_{n}^{(j)}(t)\right]}{\mathrm{d} t} \mathrm{~d} t \\
& +\pi \int_{0}^{\bar{\eta}}\left\{I_{A}(\bar{\eta})\left[\operatorname{Bi}(t)+3^{1 / 2} \operatorname{Ai}(t)\right]\right. \\
& \left.+\left[I_{B}(t) \operatorname{Ai}(t)-I_{A}(t) \operatorname{Bi}(t)\right]\right\} \frac{\mathrm{d}\left[\widehat{F}_{n}^{(j)}(t)+(n / k l) \widehat{G}_{n}^{(j)}(t)\right]}{\mathrm{d} t} \mathrm{~d} t
\end{aligned}
$$

for $j=1,2,3, \ldots$ So

and

$$
\begin{gathered}
\widehat{W}_{n}^{(1)}=-\frac{\mathrm{i} n \pi}{(\mathrm{i} k \lambda)^{2 / 3} l} \tilde{P}_{n}^{(1)}(k)\left[\mathrm{Gi}(\bar{\eta})-\frac{1}{\sqrt{3}} \operatorname{Ai}(\bar{\eta})\right], \\
\widehat{U}_{n}^{(1)}=-\frac{n}{l k} \widehat{W}_{n}^{(1)}+3 \lambda \widehat{F}_{n}(k) \int_{0}^{\bar{\eta}} \operatorname{Ai}(\tilde{\eta}) \mathrm{d} \tilde{\eta},
\end{gathered}
$$

$$
\widehat{V}_{n}^{(1)}(k)=\frac{\tilde{P}_{n}^{(1)}}{\mathrm{i} k \lambda}\left[k^{2}+(n / l)^{2}\right]+3 \mathrm{i} k \lambda \widehat{F}_{n}(k)\left[\frac{\operatorname{Ai}^{\prime}(\bar{\eta})}{(\mathrm{i} k \lambda)^{1 / 3}}-Y \int_{0}^{\bar{\eta}} \operatorname{Ai}(\tilde{\eta}) \mathrm{d} \tilde{\eta}\right],
$$


and since

$$
\operatorname{Gi}(\bar{\eta}) \sim \frac{1}{\pi \bar{\eta}}+O\left(\frac{1}{\bar{\eta}^{4}}\right)=\frac{1}{\pi(\mathrm{i} k \lambda)^{1 / 3} Y}+O\left(\frac{1}{(\mathrm{i} k \lambda)^{4 / 3} Y^{4}}\right), \quad \text { as } Y \rightarrow \infty,
$$

it follows from (5.8), (5.9) and (A 9) that

$$
\tilde{P}_{n}^{(1)}(k)=\frac{(\mathrm{i} k \lambda)^{5 / 3} 9^{1 / 3}}{\left[(n / l)^{2}+k^{2}\right] \Gamma(1 / 3)} \hat{F}_{n}(k)
$$

It now follows from (5.9), (A 7) and equation (10.4.42) of Abramowitz \& Stegun (1965) that the $n$th spanwise harmonic of the second-order wall pressure $\tilde{P}_{n}^{(2)}$ is given by $(5.21)$.

\section{Appendix B}

Our interest is in the asymptotic behaviour of the second-order wall pressure as $x \rightarrow \infty$. Equations (4.1) and (4.2) show that this quantity is determined by the asymptotic behaviour of $\tilde{P}_{n}^{(2)}$ as $k \rightarrow 0$. The appropriate formula for the latter quantity is derived in this appendix. Equations (5.16) and (5.18) show that

$$
\begin{aligned}
(\mathrm{i} k \lambda)^{5 / 3}\left[\widehat{F}_{n}^{(2)}(\bar{\eta})+(n / k l) \hat{G}_{n}^{(2)}(\bar{\eta})\right] & \\
= & -\sum_{p=-\infty}^{p=\infty} \int_{-\infty}^{\infty} \widehat{U}_{n-p}^{(1)}\left((1-\kappa)^{1 / 3} \bar{\eta}, k-k_{1}\right) \mathrm{i} k_{1} \widehat{E}_{p}^{n}\left(\kappa^{1 / 3} \bar{\eta}, k_{1}\right) \mathrm{d} k_{1} \\
& -\sum_{p=-\infty}^{p=\infty} \int_{-\infty}^{\infty} \widehat{V}_{n-p}^{(1)}\left((1-\kappa)^{1 / 3} \bar{\eta}, k-k_{1}\right)(\mathrm{i} k \lambda)^{1 / 3} \frac{\partial}{\partial \bar{\eta}} \widehat{E}_{p}^{n}\left(\kappa^{1 / 3} \bar{\eta}, k_{1}\right) \mathrm{d} k_{1} \\
& -\sum_{p=-\infty}^{p=\infty} \int_{-\infty}^{\infty} \widehat{W}_{n-p}^{(1)}\left((1-\kappa)^{1 / 3} \bar{\eta}, k-k_{1}\right) \mathrm{i}(p / l) \hat{E}_{p}^{n}\left(\kappa^{1 / 3} \bar{\eta}, k_{1}\right) \mathrm{d} k_{1},
\end{aligned}
$$

where

$$
\begin{aligned}
& \hat{E}_{p}^{n}\left(\kappa^{1 / 3} \bar{\eta}, k_{1}\right) \equiv\left\{k I_{A}\left(\bar{\eta} \kappa^{1 / 3}\right)\left[k_{1}^{2}+(p / l)^{2}\right] \frac{3^{1 / 3} \Gamma(1 / 3)}{k_{1}}\right. \\
&\left.+\left(n-\frac{p}{\kappa}\right) \frac{p \pi}{l^{2}}\left[\operatorname{Gi}\left(\kappa^{1 / 3} \bar{\eta}\right)-\frac{1}{\sqrt{3}} \operatorname{Ai}\left(\kappa^{1 / 3} \bar{\eta}\right)\right]\right\} \frac{\lambda^{2} \mathrm{i} k_{1} 9^{1 / 3} \widehat{F}_{p}\left(k_{1}\right)}{\left[(p / l)^{2}+k_{1}^{2}\right] \Gamma(1 / 3)}
\end{aligned}
$$

and

$$
\kappa \equiv k_{1} / k
$$

Then since (A 9) and (A 10) imply that

$$
\begin{aligned}
& {\left[\widehat{U}_{n-p}^{(1)}\left((1-\kappa)^{1 / 3} \bar{\eta}, k-k_{1}\right) \mathrm{i} k_{1}+\hat{V}_{n-p}^{(1)}\left((1-\kappa)^{1 / 3} \bar{\eta}, k-k_{1}\right)(\mathrm{i} k \lambda)^{1 / 3} \frac{\partial}{\partial \bar{\eta}}\right.} \\
& \left.\quad+\widehat{W}_{n-p}^{(1)}\left((1-\kappa)^{1 / 3} \bar{\eta}, k-k_{1}\right) \mathrm{i}(p / l)\right] \hat{E}_{p}^{n}\left(\kappa^{1 / 3} \bar{\eta}, k_{1}\right) \\
& \quad=\left\{\frac{\mathrm{i}\left(p k-k_{1} n\right)}{l\left(k-k_{1}\right)} \widehat{W}_{n-p}^{(1)}+\frac{\tilde{P}_{n-p}^{(1)}\left(k-k_{1}\right)}{\mathrm{i} \lambda\left(k-k_{1}\right)}\left[\left(k-k_{1}\right)^{2}+\left(\frac{n-p}{l}\right)^{2}\right](\mathrm{i} k \lambda)^{1 / 3} \frac{\partial}{\partial \bar{\eta}}\right.
\end{aligned}
$$




$$
\begin{aligned}
& +\frac{3 \mathrm{i}\left(k-k_{1}\right) \lambda \widehat{F}_{n-p}\left(k-k_{1}\right) \mathrm{Ai}^{\prime}\left((1-\kappa)^{1 / 3} \bar{\eta}\right)}{(1-\kappa)^{1 / 3}} \frac{\partial}{\partial \bar{\eta}} \\
& +3 \mathrm{i} k_{1} \lambda \widehat{F}_{n-p}\left(k-k_{1}\right) I_{A}\left(\bar{\eta}(1-\kappa)^{1 / 3}\right)\left(1+Y \frac{\mathrm{d}}{\mathrm{d} Y}\right) \\
& \left.-3 \mathrm{i} k \lambda Y \widehat{F}_{n-p}\left(k-k_{1}\right) I_{A}\left(\bar{\eta}(1-\kappa)^{1 / 3}\right)(\mathrm{i} k \lambda)^{1 / 3} \frac{\partial}{\partial \bar{\eta}}\right\} \\
& \times\left\{k I_{A}\left(\kappa^{1 / 3} \bar{\eta}\right)\left[k_{1}^{2}+(p / l)^{2}\right] \frac{3^{1 / 3} \Gamma(1 / 3)}{k_{1}}\right. \\
& \left.+\left(n-\frac{p}{\kappa}\right) \frac{p \pi}{l^{2}}\left[\mathrm{Gi}\left(\kappa^{1 / 3} \bar{\eta}\right)-\frac{1}{\sqrt{3}} \operatorname{Ai}\left(\kappa^{1 / 3} \bar{\eta}\right)\right]\right\} \frac{\mathrm{i} k_{1} \lambda^{2} 9^{1 / 3} \widehat{F}_{p}\left(k_{1}\right)}{\left[(p / l)^{2}+k_{1}^{2}\right] \Gamma(1 / 3)},
\end{aligned}
$$

(A 8), (A 11) and (A 12) show that

$$
\begin{aligned}
& {\left[\widehat{U}_{n-p}^{(1)}\left((1-\kappa)^{1 / 3} \bar{\eta}, k-k_{1}\right) \mathrm{i} k_{1}+\widehat{V}_{n-p}^{(1)}\left((1-\kappa)^{1 / 3} \bar{\eta}, k-k_{1}\right)(\mathrm{i} k \lambda)^{1 / 3} \frac{\partial}{\partial \bar{\eta}}\right.} \\
&\left.+\widehat{W}_{n-p}^{(1)}\left((1-\kappa)^{1 / 3} \bar{\eta}, k-k_{1}\right) \mathrm{i}(p / l)\right] \widehat{E}_{p}^{n}\left(\kappa^{1 / 3} \bar{\eta}, k_{1}\right) \\
& \rightarrow\left\{\frac{\mathrm{i}\left(p k-k_{1} n\right)}{l\left(k-k_{1}\right)} \widehat{W}_{n-p}^{(1)}+\frac{\tilde{P}_{n-p}^{(1)}\left(k-k_{1}\right)}{\mathrm{i} \lambda\left(k-k_{1}\right)}\left[\left(k-k_{1}\right)^{2}+\left(\frac{n-p}{l}\right)^{2}\right](\mathrm{i} k \lambda)^{1 / 3} \frac{\partial}{\partial \bar{\eta}}\right. \\
&\left.+3 \mathrm{i} k_{1} \lambda \widehat{F}_{n-p}\left(k-k_{1}\right) I_{A}\left(\bar{\eta}(1-\kappa)^{1 / 3}\right)\left(1+Y \frac{\mathrm{d}}{\mathrm{d} Y}\right)\right\} \\
& \times\left(n-\frac{p}{\kappa}\right) \frac{p \pi}{l^{2}}\left[\mathrm{Gi}\left(\kappa^{1 / 3} \bar{\eta}\right)-3^{-1 / 2} \mathrm{Ai}\left(\kappa^{1 / 3} \bar{\eta}\right)\right] \frac{\mathrm{i} k_{1} \lambda^{2} 9^{1 / 3} \widehat{F}_{p}\left(k_{1}\right)}{\left[(p / l)^{2}+k_{1}^{2}\right] \Gamma(1 / 3)}+O(k) \\
&=-\left\{\left[\mathrm{Gi}\left((1-\kappa)^{1 / 3} \bar{\eta}\right)-3^{-1 / 2} \mathrm{Ai}\left((1-\kappa)^{1 / 3} \bar{\eta}\right)\right] \frac{\left(p k-k_{1} n\right)(n-p) \pi 9^{1 / 3}}{l^{2}\left[(n / l-p / l)^{2}+\left(k-k_{1}\right)^{2}\right] \Gamma(1 / 3)}\right. \\
&\left.+\frac{\left(k-k_{1}\right)^{2 / 3} k_{1}^{1 / 3} 9^{1 / 3}}{\Gamma(1 / 3)} \frac{\partial}{\partial\left(\kappa^{1 / 3} \bar{\eta}\right)}\right\}\left(n-\frac{p}{\kappa}\right) \frac{p \pi}{l^{2}}\left[\mathrm{Gi}\left(\kappa^{1 / 3} \bar{\eta}\right)-\frac{1}{\sqrt{3}} \mathrm{Ai}\left(\kappa^{1 / 3} \bar{\eta}\right)\right] \\
& \times \frac{k_{1} \lambda^{3} 9^{1 / 3} \hat{F}_{p}\left(k_{1}\right) \hat{F}_{n-p}\left(k-k_{1}\right)}{\left[(p / l)^{2}+k_{1}^{2}\right] \Gamma(1 / 3)}+O(k),
\end{aligned}
$$

as $k \rightarrow 0$. And it follows that

$$
\begin{aligned}
\int_{0}^{\infty} & {\left[\mathrm{Gi}^{\prime}(\bar{\eta})+3^{-1 / 2} \mathrm{Ai}^{\prime}(\bar{\eta})\right]\left[\widehat{U}_{n-p}^{(1)}\left((1-\kappa)^{1 / 3} \bar{\eta}, k-k_{1}\right) \mathrm{i} k_{1}\right.} \\
& +\widehat{V}_{n-p}^{(1)}\left((1-\kappa)^{1 / 3} \bar{\eta}, k-k_{1}\right)(\mathrm{i} k \lambda)^{1 / 3} \frac{\partial}{\partial \bar{\eta}} \\
& \left.+\widehat{W}_{n-p}^{(1)}\left((1-\kappa)^{1 / 3} \bar{\eta}, k-k_{1}\right) \mathrm{i}(p / l)\right] \widehat{E}_{p}^{n}\left(\kappa^{1 / 3} \bar{\eta}, k_{1}\right) \mathrm{d} \bar{\eta} \\
\rightarrow & -\left(n-\frac{p}{\kappa}\right) \frac{p \pi}{l^{2}} \frac{k_{1} \lambda^{3} 9^{2 / 3} \hat{F}_{p}\left(k_{1}\right) \hat{F}_{n-p}\left(k-k_{1}\right)}{\left[(p / l)^{2}+\left(k_{1}\right)^{2}\right][\Gamma(1 / 3)]^{2}} \int_{0}^{\infty}\left[\mathrm{Gi}^{\prime}(\bar{\eta})\right.
\end{aligned}
$$




$$
\begin{aligned}
& \left.+3^{-1 / 2} \operatorname{Ai}^{\prime}(\bar{\eta})\right]\left\{\frac{\left(k p-k_{1} n\right)(n-p) \pi}{l^{2}\left[(n / l-p / l)^{2}+\left(k-k_{1}\right)^{2}\right]}\right. \\
& \times\left[\operatorname{Gi}\left((1-\kappa)^{1 / 3} \bar{\eta}\right)-3^{-1 / 2} \mathrm{Ai}\left((1-\kappa)^{1 / 3} \bar{\eta}\right)\right] \\
& \left.+\left(k-k_{1}\right)^{2 / 3} k_{1}^{1 / 3} \frac{\partial}{\partial\left(\kappa^{1 / 3} \bar{\eta}\right)}\right\}\left[\mathrm{Gi}\left(\kappa^{1 / 3} \bar{\eta}\right)-3^{-1 / 2} \operatorname{Ai}\left(\kappa^{1 / 3} \bar{\eta}\right)\right] \mathrm{d} \bar{\eta} \\
& \rightarrow \frac{n p \pi}{l^{2}} \frac{\lambda^{3} 9^{2 / 3} \hat{F}_{p}\left(k_{1}\right) \hat{F}_{n-p}\left(-k_{1}\right)}{\left[(p / l)^{2}+\left(k_{1}\right)^{2}\right][\Gamma(1 / 3)]^{2}}\left\{\frac{n(n-p) \pi k_{1}^{2}}{l^{2}\left[(n-p)^{2} / l^{2}+k_{1}^{2}\right]} I_{1}\left(\kappa^{1 / 3}\right)\right. \\
& \left.-\left(-k_{1}\right)^{2 / 3} k_{1}^{4 / 3} I_{2}\left(\kappa^{1 / 3}\right)\right\},
\end{aligned}
$$

as $k \rightarrow 0$, where

$$
\begin{array}{r}
I_{1}\left(\kappa^{1 / 3}\right) \equiv \int_{0}^{\infty}\left[\mathrm{Gi}^{\prime}(\bar{\eta})+\mathrm{Ai}^{\prime}(\bar{\eta}) / \sqrt{3}\right]\left[\mathrm{Gi}\left((-\kappa)^{1 / 3} \bar{\eta}\right)-3^{-1 / 2} \operatorname{Ai}\left((-\kappa)^{1 / 3} \bar{\eta}\right)\right] \\
\times\left[\mathrm{Gi}\left(\kappa^{1 / 3} \bar{\eta}\right)-3^{-1 / 2} \mathrm{Ai}\left(\kappa^{1 / 3} \bar{\eta}\right)\right] \mathrm{d} \bar{\eta} \\
I_{2}\left(\kappa^{1 / 3}\right) \equiv \int_{0}^{\infty}\left[\mathrm{Gi}^{\prime}(\bar{\eta})+3^{-1 / 2} \operatorname{Ai}^{\prime}(\bar{\eta})\right]\left[\mathrm{Gi}^{\prime}\left(\kappa^{1 / 3} \bar{\eta}\right)-3^{-1 / 2} \mathrm{Ai}^{\prime}\left(\kappa^{1 / 3} \bar{\eta}\right)\right] \mathrm{d} \bar{\eta} .
\end{array}
$$

But (A 11) and equations (10.4.4) and (10.4.4) in Abramowitz \& Stegun (1965, p. 446) show that

$$
\begin{aligned}
& I_{1}\left(\kappa^{1 / 3}\right)=\kappa^{-1 / 3} \int_{0}^{\kappa^{1 / 3} M}\left[\mathrm{Gi}^{\prime}\left(\kappa^{-1 / 3} \tilde{\eta}\right)+\mathrm{Ai}^{\prime}\left(\kappa^{-1 / 3} \tilde{\eta}\right) / 3^{1 / 2}\right] \\
& \times\left[\operatorname{Gi}\left((-1)^{1 / 3} \tilde{\eta}\right)-\frac{1}{3^{1 / 2}} \operatorname{Ai}\left((-1)^{1 / 3} \tilde{\eta}\right)\right]\left[\operatorname{Gi}(\tilde{\eta})-\frac{1}{3^{1 / 2}} \operatorname{Ai}(\tilde{\eta})\right] \mathrm{d} \tilde{\eta} \\
& +\int_{M}^{\infty}\left[\mathrm{Gi}^{\prime}(\bar{\eta})+\mathrm{Ai}^{\prime}(\bar{\eta}) / 3^{1 / 2}\right]\left[\mathrm{Gi}\left((-\kappa)^{1 / 3} \bar{\eta}\right)-\frac{1}{3^{1 / 2}} \operatorname{Ai}\left((-\kappa)^{1 / 3} \bar{\eta}\right)\right] \\
& \times\left[\operatorname{Gi}\left(\kappa^{1 / 3} \bar{\eta}\right)-\frac{1}{3^{1 / 2}} \operatorname{Ai}\left(\kappa^{1 / 3} \bar{\eta}\right)\right] \mathrm{d} \bar{\eta} \\
& \rightarrow(-\kappa)^{-1 / 3} \kappa^{-1 / 3}\left\{-\frac{(-1)^{1 / 3}}{\pi} \int_{0}^{\kappa^{1 / 3} M} \tilde{\eta}\left[\operatorname{Gi}\left((-1)^{1 / 3} \tilde{\eta}\right)-3^{-1 / 2} \operatorname{Ai}\left((-1)^{1 / 3} \tilde{\eta}\right)\right]\right. \\
& \left.\times\left[\operatorname{Gi}(\tilde{\eta})-3^{-1 / 2} \operatorname{Ai}(\tilde{\eta})\right] \mathrm{d} \tilde{\eta}+\frac{1}{\pi^{2}} \int_{M}^{\infty} \frac{\left[\mathrm{Gi}^{\prime}(\bar{\eta})+3^{-1 / 2} \mathrm{Ai}^{\prime}(\bar{\eta})\right]}{\bar{\eta}^{2}} \mathrm{~d} \bar{\eta}\right\} \\
& =(-\kappa)^{-1 / 3} \kappa^{-1 / 3}\left\{-\frac{1}{\pi} \int_{0}^{\kappa^{1 / 3} M}\left\{\tilde{\eta}(-1)^{1 / 3}\left[\mathrm{Gi}(\tilde{\eta})-3^{-1 / 2} \operatorname{Ai}(\tilde{\eta})\right]\right.\right. \\
& \left.\times\left[\operatorname{Gi}\left((-1)^{1 / 3} \tilde{\eta}\right)-3^{-1 / 2} \operatorname{Ai}\left((-1)^{1 / 3} \tilde{\eta}\right)\right]-\frac{1}{\pi^{2}(1+\tilde{\eta})}\right\} \mathrm{d} \tilde{\eta}-\frac{1}{\pi^{3}} \ln \left(1+\kappa^{1 / 3} M\right) \\
& \left.+\frac{1}{\pi^{2}} \int_{M}^{\infty}\left\{\frac{\left[\mathrm{Gi}^{\prime}(\bar{\eta})+3^{-1 / 2} \mathrm{Ai}^{\prime}(\bar{\eta})\right]}{\bar{\eta}^{2}}+\frac{1}{\pi \tilde{\eta}(1+\tilde{\eta})}\right\} \mathrm{d} \bar{\eta}-\frac{1}{\pi^{3}}[\ln (1+M)-\ln M]\right\}
\end{aligned}
$$




$$
\begin{aligned}
& \rightarrow(-\kappa)^{-1 / 3} \kappa^{-1 / 3}\left\{-\frac{1}{\pi} \int_{0}^{\kappa^{1 / 3} M}\left\{(-1)^{1 / 3} \tilde{\eta}\left[\operatorname{Gi}\left((-1)^{1 / 3} \tilde{\eta}\right)-3^{-1 / 2} \operatorname{Ai}\left((-1)^{1 / 3} \tilde{\eta}\right)\right]\right.\right. \\
& \left.\quad \times\left[\operatorname{Gi}(\tilde{\eta})-3^{-1 / 2} \operatorname{Ai}(\tilde{\eta})\right]-\frac{1}{\pi^{2}(1+\tilde{\eta})}\right\} \mathrm{d} \tilde{\eta}-\frac{1}{\pi^{3}} \ln \kappa^{1 / 3} \\
& \left.\quad+\frac{1}{\pi^{2}} \int_{M}^{\infty}\left\{\frac{\left[\mathrm{Gi}^{\prime}(\bar{\eta})+3^{-1 / 2} \mathrm{Ai}^{\prime}(\bar{\eta})\right]}{\bar{\eta}^{2}}+\frac{1}{\pi \tilde{\eta}(1+\tilde{\eta})}\right\} \mathrm{d} \bar{\eta}-\frac{1}{\pi^{3}} \ln (1+M)\right\} \text { as } k \rightarrow \infty .
\end{aligned}
$$

So

$$
\begin{aligned}
& I_{1}\left(\kappa^{1 / 3}\right) \rightarrow(-\kappa)^{-1 / 3} \kappa^{-1 / 3}\left\{-\frac{1}{\pi} \int_{0}^{\infty}\left\{(-1)^{1 / 3} \tilde{\eta}\left[\operatorname{Gi}\left((-1)^{1 / 3} \tilde{\eta}\right)-3^{-1 / 2} \operatorname{Ai}\left((-1)^{1 / 3} \tilde{\eta}\right)\right]\right.\right. \\
& \left.\quad \times\left[\operatorname{Gi}(\tilde{\eta})-3^{-1 / 2} \operatorname{Ai}(\tilde{\eta})\right]-\frac{1}{\pi^{2}(1+\tilde{\eta})}\right\} \mathrm{d} \tilde{\eta}-\frac{1}{\pi^{3}} \ln \kappa^{1 / 3} \\
& \left.\quad+\frac{1}{\pi^{2}} \int_{0}^{\infty}\left\{\frac{\left[\mathrm{Gi}^{\prime}(\bar{\eta})+3^{-1 / 2} \mathrm{Ai}^{\prime}(\bar{\eta})\right]}{\bar{\eta}^{2}}+\frac{1}{\pi \tilde{\eta}(1+\tilde{\eta})}\right\} \mathrm{d} \bar{\eta}\right\}
\end{aligned}
$$

when $M \rightarrow 0$ and $\kappa^{1 / 3} M \rightarrow \infty$, which means that

$$
I_{1}\left(\kappa^{1 / 3}\right) \rightarrow-\frac{1}{\pi^{3}(-\kappa)^{1 / 3} \kappa^{1 / 3}} \ln \kappa^{1 / 3} \quad \text { as } k \rightarrow 0 .
$$

Similarly

$$
\begin{aligned}
I_{2}\left(\kappa^{1 / 3}\right)= & \left\{\kappa^{-1 / 3} \int_{0}^{\kappa^{1 / 3} M}\left[\mathrm{Gi}^{\prime}\left(\kappa^{-1 / 3} \tilde{\eta}\right)+3^{-1 / 2} \mathrm{Ai}^{\prime}\left(\kappa^{-1 / 3} \tilde{\eta}\right)\right]\right. \\
& \times\left[\mathrm{Gi}^{\prime}(\tilde{\eta})-3^{-1 / 2} \mathrm{Ai}^{\prime}(\tilde{\eta})\right] \mathrm{d} \tilde{\eta}+\int_{M}^{\infty}\left[\mathrm{Gi}^{\prime}(\bar{\eta})+3^{-1 / 2} \mathrm{Ai}^{\prime}(\bar{\eta})\right] \\
& \left.\times\left[\mathrm{Gi}^{\prime}\left(\kappa^{1 / 3} \bar{\eta}\right)-3^{-1 / 2} \mathrm{Ai}^{\prime}\left(\kappa^{1 / 3} \bar{\eta}\right)\right] \mathrm{d} \bar{\eta}\right\} \\
\rightarrow & -\kappa^{-2 / 3} \frac{1}{\pi}\left\{\int_{0}^{\kappa^{1 / 3} M} \tilde{\eta}\left[\mathrm{Gi}^{\prime}(\tilde{\eta})-3^{-1 / 2} \mathrm{Ai}^{\prime}(\tilde{\eta})\right] \mathrm{d} \tilde{\eta}+\int_{M}^{\infty} \frac{\left[\mathrm{Gi}^{\prime}(\bar{\eta})+3^{-1 / 2} \mathrm{Ai}^{\prime}(\bar{\eta})\right]}{\bar{\eta}^{2}} \mathrm{~d} \bar{\eta}\right\} \\
= & -\kappa^{-2 / 3} \frac{1}{\pi}\left\{\int_{0}^{\kappa^{1 / 3} M}\left\{\tilde{\eta}\left[\mathrm{Gi}^{\prime}(\tilde{\eta})-3^{-1 / 2} \mathrm{Ai}^{\prime}(\tilde{\eta})\right]+\frac{1}{\pi(1+\tilde{\eta})}\right\} \mathrm{d} \tilde{\eta}-\frac{1}{\pi} \ln \left(1+\kappa^{1 / 3} M\right)\right. \\
& \left.+\int_{M}^{\infty}\left\{\frac{\left[\mathrm{Gi}^{\prime}(\bar{\eta})+3^{-1 / 2} \mathrm{Ai}^{\prime}(\bar{\eta})\right]}{\bar{\eta}^{2}}+\frac{1}{\pi \bar{\eta}(1+\bar{\eta})}\right\} \mathrm{d} \bar{\eta}-\frac{1}{\pi}[\ln (1+M)-\ln M]\right\} \\
\rightarrow & \frac{1}{\pi^{2} \kappa^{2 / 3}} \ln \kappa^{1 / 3} .
\end{aligned}
$$

And so

$$
\begin{aligned}
& \int_{0}^{\infty}\left[\mathrm{Gi}^{\prime}(\bar{\eta})+\mathrm{Ai}^{\prime}(\bar{\eta}) / \sqrt{3}\right]\left[\widehat{U}_{n-p}^{(1)}\left((1-\kappa)^{1 / 3} \bar{\eta}, k-k_{1}\right) \mathrm{i} k_{1}\right. \\
& \left.\quad+\widehat{V}_{n-p}^{(1)}\left((1-\kappa)^{1 / 3} \bar{\eta}, k-k_{1}\right)(\mathrm{i} k \lambda)^{1 / 3} \frac{\partial}{\partial \bar{\eta}}+\widehat{W}_{n-p}^{(1)}\left((1-\kappa)^{1 / 3} \bar{\eta}, k-k_{1}\right) \mathrm{i}(p / l)\right]
\end{aligned}
$$




$$
\begin{aligned}
& \times \widehat{E}_{p}^{n}\left(\kappa^{1 / 3} \bar{\eta}, k_{1}\right) \mathrm{d} \bar{\eta} \rightarrow \frac{n p \pi \lambda^{3} 9^{2 / 3} \hat{F}_{p}\left(k_{1}\right) \hat{F}_{n-p}\left(-k_{1}\right)}{\left[p^{2}+\left(l k_{1}\right)^{2}\right][\Gamma(1 / 3)]^{2}} \\
& \times\left\{\frac{n(n-p)}{\left[(n-p)^{2}+k_{1}^{2} l^{2}\right]}-1\right\} \frac{\left(-k_{1}\right)^{2 / 3} k_{1}^{2 / 3} k^{2 / 3}}{\pi^{2}} \ln \kappa^{1 / 3} \quad \text { as } k \rightarrow 0 .
\end{aligned}
$$

Equations (B1) and (B 13) now show that the asymptotic behaviour of the $n$th spanwise harmonic of the second-order wall pressure $\tilde{P}_{n}^{(2)}$ as $k \rightarrow 0$ is given by $(5.22)$.

\section{REFERENCES}

Abramowitz, M. \& Stegun, I. A. 1965 Handbook of Mathematical Functions, National Bureau of Standards.

ACarlar, M. S. \& Smith, C. R. 1987 A study of hairpin vortices in a laminar boundary layer. Part 1. J. Fluid Mech. 175, 1-42.

Andersson, P., Berggren, M. \& Henningson, D. S. 1999 Optimal disturbances and bypass transition in boundary layers. Phys. Fluids 11 (1), 134-150.

Bender, C. M. \& Orszag, S. A. 1978 Advanced Mathematical Methods for Scientists and Engineers. McGraw-Hill.

Carrier, G. F., Krook, M. \& Pearson, C. E. 1966 Functions of a Complex Variable. McGraw-Hill.

CASE, K. M. 1960 Stability of plane Couette flow. Phys. Fluids 3, 143-148.

Choudhari, M. \& Duck, P. W. 1996 Nonlinear excitation of inviscid stationary vortex instabilities in boundary layer flow. In Proceedings of the IUTAM Symposium on Nonlinear Instability and Transition in Three-dimensional Boundary Layers (ed. P. W. Duck and P. Hall), pp. 409-422 Manchester, UK, 17-20 July 1995. Kluwer.

Choudhari, M. \& Fischer, P. 2005 Roughness induced transient growth. In Proceedings of the 35th AIAA Fluid Dynamics Conference and Exhibition. AIAA Paper 2005-4765.

Ellingson, T. \& Palm, E. 1975 Stability of linear flow. Phys. Fluids 18, 487-488.

Fischer, P. \& Choudhari, M. 2004 Numerical simulation of roughness-induced transient growth in a laminar boundary layer. AIAA Paper 2004-2539.

Fransson, J. M. H., Brant, L., Talamelli, A. \& Cossu, C. 2004 Experimental and theoretical investigation of non-modal growth of steady streaks in a flat plate boundary layer. Phys. Fluids 16, 3627.

Fransson, J. M. H., Talamelli, A., Brant, L. \& Cossu, C. 2006 Delaying transition to turbulence by a passive mechanism. Phys. Rev. Lett. 96, 060541.

Goldstein, M. E., Sescu, A., Duck, P. W. \& Choudhari, M. 2010 The long range persistence of wakes behind a row of roughness elements. J. Fluid Mech. 644, 123-163.

Kemp, N. H. 1951 The laminar three-dimensional boundary layer and the study of the flow past a side edge. MAeS thesis, Cornell University.

Klebanoff, P., Cleveland, W. G. \& Tidstrom, K. D. 1992 On the evolution of a turbulent boundary layer induced by a three-dimensional roughness element. J. Fluid Mech. 237, 101-187.

LANDAhL, M. T. 1980 A note on an algebraic instability of inviscid parallel shear flows. J. Fluid Mech. 98, 243-251.

LUCHINI, P. 2000 Reynolds-number-independent instability of the boundary layer over a flat surface: optimal perturbations. J. Fluid Mech. 404, 289-309.

RothmaYeR, A. P. \& Smith, F. T. 1998 Incompressible triple deck theory. In The Handbook of Fluid Dynamics, chap. 23, CRC.

Smith, F. T. 1973 Laminar flow over a small hump on a flat plate. J. Fluid Mech. 57, 803-824.

Sмith, F. T. 1976 a Flow through constricted pipes and channels. Part 1. Q. J. Mech. Appl. Math. 29, 343-364.

Smith, F. T. $1976 b$ Flow through constricted pipes and channels. Part 2. Q. J. Mech. Appl. Math. 29, 365-376. 
Smith, F. T., Brighton, P. S., Jackson, P. S. \& Hunt, J. C. R. 1981 On boundary layer flow over two dimensional obstacles. J. Fluid Mech. 113, 123-152.

Tumin, A. \& Reshotko, E. 2001 Spatial theory of optimal disturbances in boundary layers. Phys. Fluids 13 (7), 2097-2104.

Van Dyke, M. 1975 Perturbation Methods in Fluid Mechanics. The Parabolic Press.

White, E. B. \& Ergin, F. G. 2003 Receptivity and transient growth of roughness-induced disturbances. AIAA Paper 2003-4243. 